\title{
¿On Tidal Modulation of the Evolution of Internal Solitary-Like Waves Passing through a Critical Point
}

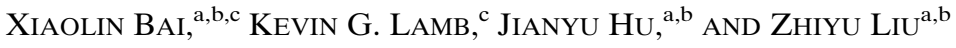

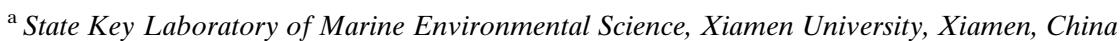 \\ ${ }^{\mathrm{b}}$ Department of Physical Oceanography, College of Ocean and Earth Sciences, Xiamen University, \\ Xiamen, China \\ ${ }^{\mathrm{c}}$ Department of Applied Mathematics, University of Waterloo, Waterloo, Canada
}

(Manuscript received 28 July 2020, in final form 3 April 2021)

\begin{abstract}
Internal solitary-like waves (ISWs) evolve considerably when passing through a critical point separating the deep water where ISWs are waves of depression and shallower water where they are waves of elevation. The location of the critical point is determined by the background current and stratification. In this study, we investigate the influence of tidal currents on the cross-shelf movement of the critical point and elucidate the underlying processes via fully nonlinear numerical simulations. Our simulations reveal phase-locked tidal variations of the critical point, which are mainly attributed to stratification fluctuations that are modulated by the combined effects of cross-shelf barotropic tidal currents and locally generated baroclinic tides. The barotropic tidal currents drive isopycnal displacements as they flow over the slope, and as this occurs baroclinic tides are generated, modulating the stratification and inducing sheared currents. This results in a crossshelf movement of the critical point, which moves onshore (offshore) when the pycnocline is elevated (depressed) by the flood (ebb) tide. Our idealized numerical simulations for the study region in the South China Sea suggest that the cross-shelf movement of the critical point reaches to $O(10) \mathrm{km}$ within a tidal cycle. This distance depends on the strength of tidal currents, stratification, and bathymetry. Because of tidal currents, ISWs of depression may undergo a complex evolution even in a stratification with a shallow pycnocline. For the stratification with a deep pycnocline, the critical point may be at a location deep enough so that its tidal movement becomes insignificant.
\end{abstract}

KEYWORDS: Internal waves; Solitary waves; Tides

\section{Introduction}

Internal solitary-like waves (ISWs) and undular bores are common features of the coastal ocean (e.g., Grimshaw et al. 2011; Jackson and Apel 2004). ISWs, which usually appear in packets, are often well approximated by solutions of the Dubreil-Jacotin-Long (DJL) equation, which gives exact solitary wave solutions of the incompressible Euler equations in a nonrotating, inviscid, horizontally homogeneous ocean with a rigid lid (Lamb 2002; Stastna and Lamb 2002). Mathematically idealized solitary waves propagate without changing form, but ISWs in the ocean gradually lose energy due to a number of processes. These include shoaling, which induces wave reflections and may result in fissioning whereby an initial single wave evolves into a number of waves, rotationally induced radiation of inertia-gravity waves, interactions with surface waves, viscous effects, and transfer of energy to other waves via nonlinear interactions (Bai et al. 2017, 2019; Gargettt and Hughes 1972; Helfrich and Grimshaw 2008; Lamb 2004; Lamb and Warn-Varnas 2015; Staquet and Sommeria 2002). When these processes affect the waves on long time scales, which is often

\footnotetext{
D Denotes content that is immediately available upon publication as open access.
}

Corresponding authors: Zhiyu Liu, zyliu@xmu.edu.cn; Jianyu Hu, hujy@xmu.edu.cn the case, the DJL solutions can provide a good description of the instantaneous shape and propagation speed of the waves.

ISWs are typically waves of depression/elevation (referred to as the polarity of the waves) in sufficiently deep/shallow water. A simple example of this is provided by a stratification consisting of two layers of constant density fluid, both at rest. Under the Boussinesq approximation ISWs are waves of depression/elevation when the interface is in the upper/lower half of the water column. These waves have a maximum amplitude given by the distance from the interface to the middepth (Lamb and Xiao 2014). For a continuous stratification with a single thin pycnocline above and below which the density is very weakly stratified, e.g., a hyperbolic tangent density profile, ISWs are again waves of depression/elevation if the pycnocline is above/below the middepth although now the distance to the middepth only approximately provides the limiting amplitude.

Here we are interested in the evolution of a shoaling ISW as it passes through the region where DJL solitary waves are waves of depression to a shallower region where they are waves of elevation. The location at which the polarity of ISWs changes sign is called the critical point. As the critical point is approached from shallower or deeper water the maximum possible wave amplitude (in absolute value) goes to zero. Returning to the two-layer example taken above, as an ISW of depression approaches a critical point, in this case located where the upper and lower layers have identical thicknesses, from deep water the limiting ISW amplitude goes to zero. Thus, at some location the wave amplitude will be larger 
than the limiting wave amplitude and the wave will be strongly affected by the changing water depth, possibly well before the critical point is reached. This results in the wave becoming asymmetrical as the leading half of the wave becomes less steep and the interface is pushed above its undisturbed height behind the wave. The details of this process and the degree of deformation depends on how rapidly the water depth changes, in particular on the ratio of the time scale of the depth changes to the adjustment time scale for the wave as it shoals (e.g., Lamb and Warn-Varnas 2015).

The end result of an initial ISW of depression passing through a critical point is the transformation of the wave into a mix of waves of elevation and depression, a process which starts well before the critical point is reached unless the bathymetry is very steep. On the shallow side of the critical point ISWs of elevation often emerge, a process that is sometimes referred to as polarity conversion or polarity reversal (Aghsaee et al. 2010; Grimshaw et al. 2010; Li et al. 2015; Liu et al. 1998; Orr and Mignerey 2003; Shroyer et al. 2009; Zhao et al. 2003, 2004). The formation of ISWs of elevation has been observed from variations of isothermal and isopycnal as well as acoustic backscatter (Fu et al. 2012; Orr and Mignerey 2003; Ramp et al. 2004; Shroyer et al. 2009; X. Zhang et al. 2018), and in numerical simulations (Grimshaw et al. 2004; Lamb and Xiao 2014; Liu et al. 1998). It is impossible for a single wave of depression to be converted solely into one or more waves of elevation by consideration of conservation of mass which suggests that waves of depression must dominate the wave field in shallow water in the sense that the integrated vertical displacement of all isopycnals must remain negative for all time.

The formation of ISWs of elevation as ISWs of depression or undular bores pass through a critical point has also been observed by high-resolution satellite images which detect changes in sea surface roughness that are induced by ISW induced surface currents (Liu et al. 1998; Zhao et al. 2003, 2004). Passing through a critical point plays an essential role in the evolution of ISWs, including dramatic changes in waveform, wave amplitude and vertical structure of the wave-induced velocity (e.g., Liu et al. 1998; Orr and Mignerey 2003; Zhao et al. 2003; Ramp et al. 2004; Shroyer et al. 2009; Bai et al. 2019). The resulting waves of elevation lift nutrient-enriched and high-chlorophyll subsurface water into the euphotic zone where phytoplankton can be exposed to high light levels (e.g., Sandstrom and Elliott 1984; Woodson 2018). Moreover, Orr and Mignerey (2003) observed high turbulent dissipation rates in the vicinity of the critical point. As such, knowledge of its location is crucial to predicting the impacts of ISWs on ocean mixing and marine ecology.

In recent years, research efforts have been devoted to the study of critical point variations due to the seasonality of stratification and mesoscale oceanic motions, for example in the South China Sea (SCS) (Cai et al. 2014; Liao et al. 2014; S. Zhang et al. 2018; X. Zhang et al. 2018) and in the Sea of Okhotsk (Kurkina et al. 2017). However, the variations of the stratification on the time scale of shoaling ISWs, i.e., $O(1)$ day, have been commonly assumed to have a negligible effect on the location of the critical point, and hence ISW polarity is usually assumed not to vary with time at a particular location (Osborne and Burch 1980; Stanton and Ostrovsky 1998; Klymak and Moum 2003; Scotti and Pineda 2004; Shroyer et al. 2009). Consequently, the effects of tidal currents, especially barotropic tidal currents, on shoaling ISWs have attracted little attention, although they have been shown to modulate the propagation speed and wavelength of ISWs (Fan et al. 2013). Moreover, our knowledge on the spatial-temporal variations of the critical point due to tides remains rather limited. Recent studies in Laoshan Bay (Li et al. 2015; Grimshaw et al. 2016) reporting tide-induced ISW polarity conversion are important exceptions. There, the observations were from a shallow site with a mean depth of $9 \mathrm{~m}$, where the pycnocline depth relative to the total water depth is significantly modulated by tidal variations of the water depth. Motivated by these studies, we seek to evaluate the spatial-temporal variations of the evolution of ISWs propagating toward and through a critical point whose location varies on tidal scales. The results are crucial to understanding the dynamical evolution of shoaling ISWs, and to improving ISW predictions in complex ocean environments.

To this end, we focus on the influence of tidal currents on the evolution of ISWs shoaling through a critical point in a twodimensional setting with a horizontally uniform stratification. We will show that the location of the critical point can vary substantially over a tidal period. Unlike the case in Laoshan Bay, we consider a continental shelf region where tidal elevations are much smaller than the mean water depth, and where changes in stratification and background currents are significant due to the interaction of the barotropic tides with the sloping bottom. Our investigation is based on the northeastern SCS (Fig. 1a) using a fully nonlinear numerical model. The SCS is an excellent natural laboratory for internal wave studies (e.g., Alford et al. 2015), but the results reported here are expected to be applicable to other shelf regions of the world's oceans.

\section{Theoretical considerations}

\section{a. Weakly nonlinear theory}

The critical point is usually defined as the location where the coefficient of the quadratic nonlinear term in the Korteweg-de Vries (KdV) equation changes sign (Grimshaw et al. 2004; Shroyer et al. 2009; Cai et al. 2014; Liao et al. 2014; S. Zhang et al. 2018) although a more accurate determination requires the use of conjugate flow solutions or DJL solutions. For some stratifications ISWs of both polarities exist even when the quadratic coefficient is zero (Talipova et al. 1999). In a continuously stratified flow, the quadratic nonlinear coefficient is

$$
\alpha=\frac{3}{2} \frac{\int_{-H}^{0}(c-U)^{2}(\partial \phi / \partial z)^{3} d z}{\int_{-H}^{0}(c-U)(\partial \phi / \partial z)^{2} d z},
$$

where $c$ is the phase speed of linear long waves, $U(z)$ is the background current in the wave propagation direction, and $\phi(z)$ is the mode- 1 eigenfunction (e.g., Shroyer et al. 2009; Li et al. 2015; Grimshaw et al. 2016). Our discussion will be 


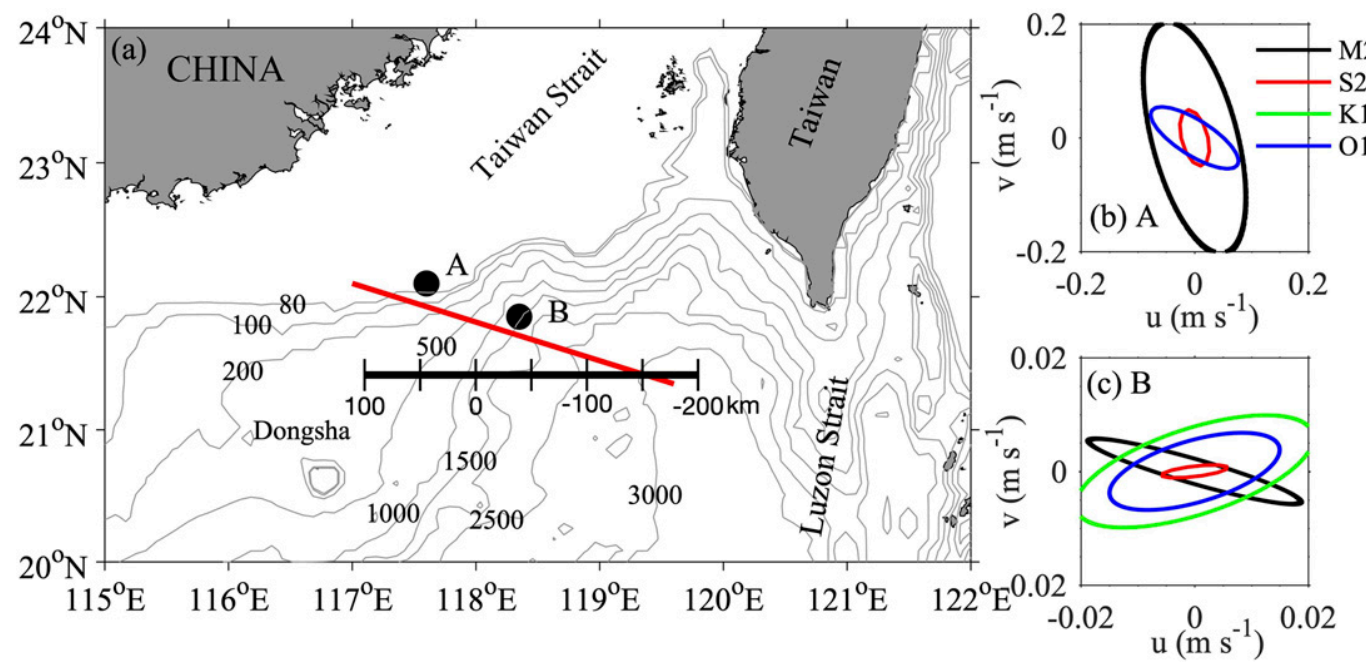

FIG. 1. (a) Bathymetry in the northern South China Sea. The red line indicates the shoaling path of ISWs. Tidal ellipses of the four main tidal constituents $\left(\mathrm{K}_{1}, \mathrm{O}_{1}, \mathrm{M}_{2}\right.$, and $\left.\mathrm{S}_{2}\right)$ at (b) station A and (c) station B are shown. Tidal ellipse of $\mathrm{O}_{1}$ is superimposed on that of $\mathrm{K}_{1}$ so that no green ellipse is shown in (b).

limited to the case where $c$ is everywhere greater than $U$. In a two-layer flow under the Boussinesq approximation,

$$
\alpha=\frac{3}{2}\left(\frac{c-U_{2}}{h_{2}}-\frac{c-U_{1}}{h_{1}}\right),
$$

where the upper (lower) layer has a thickness of $h_{1}\left(h_{2}\right)$ and a vertically uniform background current $U_{1}\left(U_{2}\right)$. According to Grimshaw et al. (2016), the phase speed of linear long waves in the two-layer model is

$c=U-\frac{h_{1}-h_{2}}{h_{1}+h_{2}} \Delta U \pm \frac{1}{h_{1}+h_{2}} \sqrt{g^{\prime} h_{1} h_{2}\left(h_{1}+h_{2}\right)-4 h_{1} h_{2}(\Delta U)^{2}}$,

where $U$ is the depth-averaged current speed, $\Delta U=0.5\left(U_{1}-\right.$ $\left.U_{2}\right)$, and $g^{\prime}=\left[\left(\rho_{2}-\rho_{1}\right) / \rho_{2}\right] g$ is the reduced gravity with $\rho_{1}$ and $\rho_{2}$ being the density in the upper and lower layers, respectively. As such, $\alpha$ is determined by the stratification and the vertical shear (here simply the velocity difference in a two-layer setting) of the background current $\Delta U$. When $\Delta U=0$ the critical point is at the location where $h_{1}=h_{2}$. In a background current with vertical shear, the critical point is shifted to deeper water when $U_{1}>U_{2}$, and to shallower water when $U_{1}<U_{2}$.

In a steady background current and stratification, predicting the location of the critical point is straightforward using Eq. (1). Its simplification in a two-layer flow is also commonly used to estimate the location of the critical point for steady flows.

\section{b. Parameter space}

The evolution of shoaling ISWs in the presence of tidal currents has a rich parameter space even in a two-dimensional setting with a horizontally uniform stratification. It depends on details of the stratification, background currents and bathymetry, as well as on the amplitude of the various tidal constituents and on the Coriolis frequency. In a two-dimensional setting, the simplest scenario is possibly that of a shoaling ISW in a two-layer stratification with a constant bottom slope and a single tidal constituent. Then the location of the temporally varying critical point depends on the depth of the interface, the strength of the tidal current, the tidal and Coriolis frequencies, and the bottom slope. The transformation of the ISW as it propagates through the critical point will depend on its initial amplitude and the phase of the tidal current at the time the critical point is reached.

A simple estimate of the location of the critical point can be obtained by assuming the water is advected only by the barotropic tidal currents (i.e., ignoring baroclinic effects which modify the stratification and introduce baroclinic currents). Because the horizontal barotropic current is vertically uniform and the vertical velocity varies linearly with depth, the water column is advected with no tilting, and it is uniformly stretched/compressed as it moves into deeper/shallower water. Consequently, the vertical eigenfunction is stretched/compressed likewise. This means that the quadratic coefficient $\alpha$ is multiplied by a positive constant and hence is zero if and only if it was initially zero. In other words, the critical point is simply advected by the barotropic tidal current. Let $x_{c}$ be the position of the critical point in the undisturbed fluid (e.g., at peak flood or ebb tide or at $t=0$ in our setup) and $x(t)$ be its position at time $t$ then we have

$$
\frac{d x}{d t}=\frac{Q_{0}}{H[x(t)]} \cos (\omega t)
$$

where $Q_{0}$ is the peak volume flux and $H(x)$ is the water depth. For a linear bottom slope, $H(x)=H_{c}-s\left(x-x_{c}\right)$, where $H_{c}$ is the water depth at $x_{c}$ and $s$ is the constant bottom slope, and we can integrate Eq. (4) to obtain

$$
\frac{s}{2}\left(x-x_{c}\right)^{2}-H_{c}\left(x-x_{c}\right)+\frac{Q_{0}}{\omega} \sin (\omega t)=0 .
$$




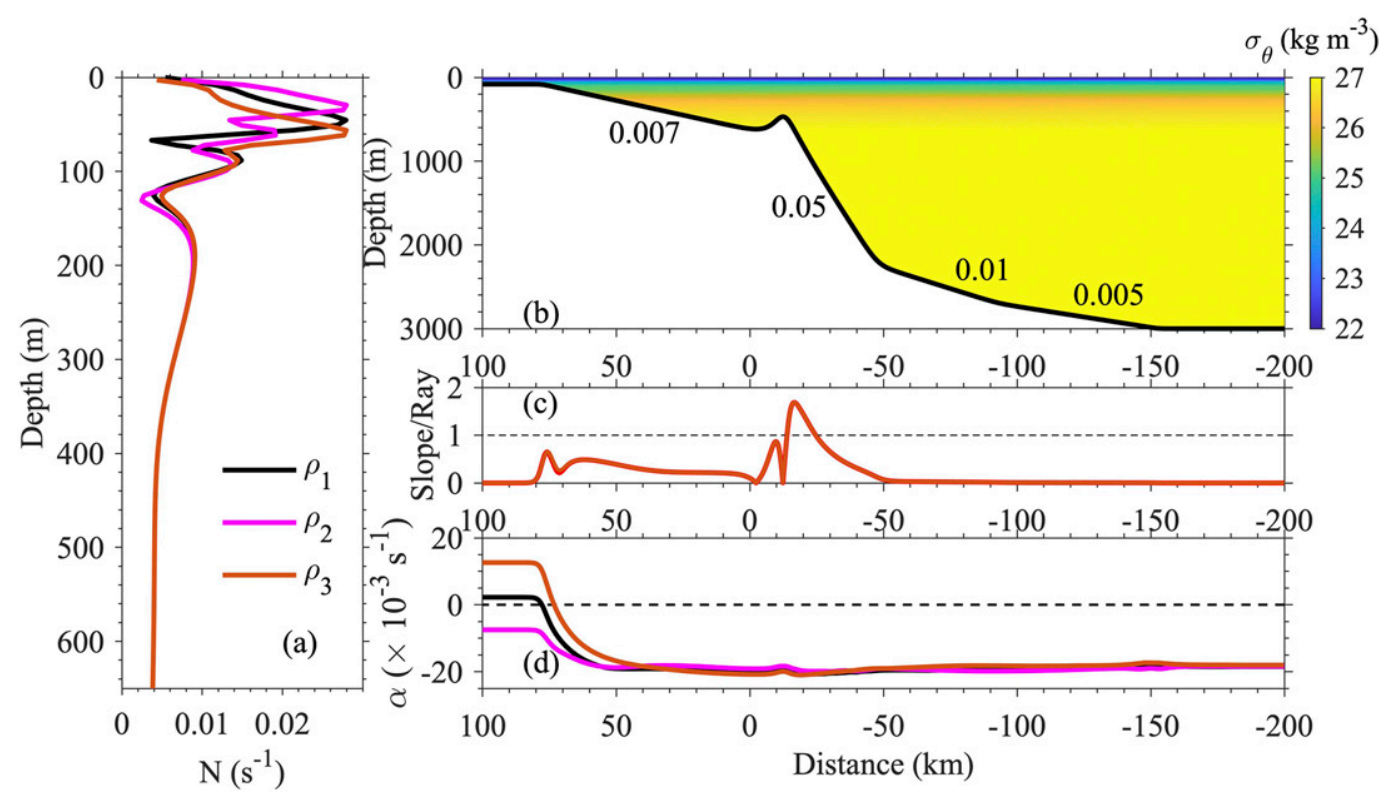

FIG. 2. (a) Three profiles of the buoyancy frequency $N\left(\mathrm{rad} \mathrm{s}^{-1}\right)$ used in the numerical simulations. (b) Bathymetry along ISW propagation path (red line in Fig. 1a) and the background density field $\left(\sigma_{\theta}\right)$. The numbers show slopes of the bathymetry. (c) Slope criticality for the $\mathrm{M}_{2}$ tide for the three stratifications. The dashed line, i.e., criticality equals to 1 , distinguishes the regions of subcritical and supercritical slope. (d) The corresponding quadratic nonlinear coefficient $\alpha$ over the study domain.

Solving the above quadratic equation, we have approximate peak displacements at the end of flood [taking $\sin (\omega t)=1$ ] and ebb [taking $\sin (\omega t)=-1$ ] tides as

$$
\begin{aligned}
& \Delta x_{f}=\frac{Q_{0}}{\omega H_{c}}+\frac{Q_{0}^{2}}{\omega^{2} H_{c}^{3}} s=\frac{U_{c}}{\omega}+\frac{U_{c}^{2}}{\omega^{2}} \frac{s}{H_{c}}, \\
& \Delta x_{e}=\frac{Q_{0}}{\omega H_{c}}-\frac{Q_{0}^{2}}{\omega^{2} H_{c}^{3}} s=\frac{U_{c}}{\omega}-\frac{U_{c}^{2}}{\omega^{2}} \frac{s}{H_{c}},
\end{aligned}
$$

Note that $U_{c} / \omega$ is the tidal excursion for a flat bottom. Here we use the fact that at peak tidal current $\left[2 s Q_{0} /\left(\omega H_{c}^{2}\right)\right] \sin (\omega t) \approx 0.373$ for our base simulations $\left(0.3 \mathrm{~m} \mathrm{~s}^{-1}\right.$ at $80-\mathrm{m}$ depth so $Q_{0}=24 \mathrm{~m}^{2} \mathrm{~s}^{-1}$, $\omega=1.4075 \times 10^{-4} \mathrm{~s}^{-1}$ and $\left.s=0.007\right)$ and use the first three terms of the Taylor series expansion of the radical above.

These results suggest that to the leading order in $\left(U_{c} / \omega\right)\left(s / H_{c}\right)$ (the ratio of the tidal excursion $U_{c} / \omega$ to the water depth at the critical point times the bottom slope) the critical point moves a distance equal to the tidal excursion for a flat bottom with a small increase (decrease) for flood (ebb) tide because of the increase (decrease) in the barotropic current as fluid moves into shallower (deeper) water. This small correction is linear with respect to the bottom slope.

\section{Modeling}

\section{a. Model description}

We conduct process-oriented numerical simulations using a fully nonlinear, two-dimensional nonhydrostatic internal gravity wave model (Lamb 1994, 2007; Lamb and Warn-Varnas 2015; Bai et al. 2017). The model uses a second-order, finite volume projection method with Godunov flux limiting which acts as an implicit large-eddy simulation filtering (Bell and Marcus 1992; Margolin et al. 2006; Lamb and Dunphy 2018). It uses terrain-following coordinates and the rigid-lid, Boussinesq, and $f$-plane approximations. The governing equations are

$$
\begin{aligned}
\mathbf{u}_{t}+\mathbf{u} \cdot \nabla \mathbf{u}-f \mathbf{u} \times \hat{k} & =-\frac{1}{\rho_{0}} \nabla p-\frac{\rho}{\rho_{0}} g \hat{k}+\nu \nabla^{2} \mathbf{u}, \\
\rho_{t}+\mathbf{u} \cdot \nabla \rho & =\kappa \nabla^{2} \rho, \\
\nabla \cdot \mathbf{u} & =0
\end{aligned}
$$

where $\mathbf{u}=(u, v, w)$ is the velocity vector, $(x, z)$ the cross-shore and vertical coordinates, $y$ the along-shore coordinate, $p$ is the pressure, and $g$ is the gravitational acceleration. And $\rho$ is the density, a reference value of which is $\rho_{0}$. The Coriolis parameter is taken to be $f=5.33 \times 10^{-5} \mathrm{~s}^{-1}$ corresponding to the latitude $21.5^{\circ} \mathrm{N}$ (with the northeastern SCS in mind), and both $\nu$ and $\kappa$ are set to zero in this study because of their small influence according to our sensitivity experiments (Bai et al. 2019). The use of a two-dimensional model eliminates potentially important three-dimensional effects such as refraction but simplifies the simulations and the analysis.

\section{b. Model settings}

The stratification profile $\rho_{1}$ (Fig. 2a) used in the simulations is obtained from recent Argo float measurements in the northeastern SCS (Bai et al. 2017), with the maximum buoyancy frequency layer at $45.5-\mathrm{m}$ depth. Stratification profile $\rho_{2}$ has a shallower pycnocline at $30-\mathrm{m}$ depth and $\rho_{3}$ has a deeper pycnocline at $60-\mathrm{m}$ depth. Stratification profiles $\rho_{2}$ and $\rho_{3}$ are 
TABLE 1. Parameters for the numerical simulations; $U_{0}$ is the tidal current amplitude at the shallow water boundary (depth $80 \mathrm{~m}$ ).

\begin{tabular}{|c|c|c|c|c|}
\hline Expt & Density & $U_{0}\left(\mathrm{~m} \mathrm{~s}^{-1}\right)$ & $\varphi$ & Short description \\
\hline Case 1 & $\rho_{1}$ & 0 & 0 & No tidal current; the slope is 0.007 in the upper $600 \mathrm{~m}$ \\
\hline Case 2 & $\rho_{1}$ & 0.3 & $\pi / 2$ & ISW approaches a nominal critical point during a flood tide \\
\hline Case 3 & $\rho_{1}$ & 0.3 & $3 \pi / 2$ & ISW approaches a nominal critical point during an ebb tide \\
\hline Case $1 \mathrm{~L}$ & $\rho_{1}$ & 0 & 0 & Same as Cases $1-3$, but for larger-amplitude ISW at the initial \\
\hline Case $2 \mathrm{~L}$ & $\rho_{1}$ & 0.3 & $\pi / 2$ & time $(45 \mathrm{~m})$. Wave amplitude is $25 \mathrm{~m}$ in other cases. \\
\hline Case $3 \mathrm{~L}$ & $\rho_{1}$ & 0.3 & $3 \pi / 2$ & \\
\hline Case $2 \mathrm{~g}$ & $\rho_{1}$ & 0.3 & $\pi / 2$ & Same as Case 2 but without gravity \\
\hline Case 4 & $\rho_{1}$ & 0.45 & $\pi / 2$ & Larger tidal current \\
\hline Case 5 & $\rho_{1}$ & 0.19 & $\pi / 2$ & Smaller tidal current \\
\hline Case 6 & $\rho_{2}$ & 0 & 0 & Shallower pycnocline $\rho_{2}$ \\
\hline Case 7 & $\rho_{2}$ & 0.3 & $\pi / 2$ & \\
\hline Case 8 & $\rho_{2}$ & 0.3 & $3 \pi / 2$ & \\
\hline Case 9 & $\rho_{3}$ & 0 & 0 & Deeper pycnocline $\rho_{3}$ \\
\hline Case 10 & $\rho_{3}$ & 0.3 & $\pi / 2$ & \\
\hline Case 11 & $\rho_{3}$ & 0.3 & $3 \pi / 2$ & \\
\hline Case 12 & $\rho_{1}$ & 0.3 & $\pi / 2$ & Same as Case 2, but without the bump at $x=-12.5 \mathrm{~km}$ \\
\hline Case 13 & $\rho_{1}$ & 0.3 & $\pi / 2$ & $\begin{array}{l}\text { Same as Case 2, but with a gentle slope (0.005) in the } \\
\text { upper } 600 \mathrm{~m}\end{array}$ \\
\hline Case 14 & $\rho_{1}$ & 0.3 & $\pi / 2$ & $\begin{array}{l}\text { Same as Case 2, but with a steep slope (0.009) in the } \\
\text { upper } 600 \mathrm{~m}\end{array}$ \\
\hline Case 15 & $\rho_{1}$ & 0.3 & $\pi / 2$ & Same as Case 13 , but without the bump at $x=-12.5 \mathrm{~km}$ \\
\hline Case 16 & $\rho_{1}$ & 0.3 & $\pi / 2$ & Same as Case 14 , but without the bump at $x=-12.5 \mathrm{~km}$ \\
\hline
\end{tabular}

employed to test the sensitivity of tidal modulation to stratification. Profile $\rho_{2}$ is a fit to measurements at the Asian Seas International Acoustic Experiment (ASIAEX) site (Orr and Mignerey 2003), which was used by Lamb and Warn-Varnas (2015). The background stratification is taken to be horizontally uniform in our simulations (Fig. 2b). Figure $2 b$ also shows the topography along the shoaling path of ISWs indicated in Fig. 1a, which is extracted from the digital bathymetry 2-min resolution (DB2) database. The shallow shelf starts near $x=$ $80 \mathrm{~km}$. The domain of the simulations is sufficiently large (between -966 and $634 \mathrm{~km}$ ) so that no waves reach the open boundary by the end of the simulations.

In the absence of a background current, the quadratic nonlinear coefficient $\alpha$ depends only on stratification (Fig. 2d). For stratifications $\rho_{1}$ and $\rho_{3}$, the nominal critical points, defined as the location where $\alpha=0$ when the fluid is at rest, are located at $x=77.8 \mathrm{~km}$ (depth is $85.0 \mathrm{~m}$ ) and $73.3 \mathrm{~km}$ (depth is $111.9 \mathrm{~m}$ ), respectively. There is no critical point for stratification $\rho_{2}$ which has the shallowest pycnocline.

Following Lamb and Warn-Varnas (2015), initial ISWs with a desired amplitude are computed by solving the DJL equation. For this study, initial ISWs with amplitudes of 25 and $45 \mathrm{~m}$ are used. They are located at the base of the continental slope at $x=-150 \mathrm{~km}$ in a water of $3000 \mathrm{~m}$ deep. They propagate approximately $230 \mathrm{~km}$ over approximately 1.5 days before reaching the critical point. ISWs with such amplitudes are typical of the world's oceans and were frequently observed near the ASIAEX region in the northeastern SCS (e.g., Liu et al. 2004; Zhao et al. 2004; Bai et al. 2019; Ramp et al. 2019). Although observations of ISWs with amplitudes of $100 \mathrm{~m}$ are common in field measurements in the SCS (Klymak et al. 2006; Lien et al. 2014; Huang et al. 2016), simulations with such large-amplitude ISWs are not presented in this paper because such large waves are not typical in the world's oceans and are prone to more violent breakup as they shoal and hence are more difficult to simulate. While ISWs often appear as wave packets or at the front of an undular bore, we consider the evolution of a single ISW for simplicity.

We set up the model using 12000 grid cells in the horizontal direction with a resolution of $20 \mathrm{~m}$ in the main domain (between -200 and $100 \mathrm{~km}$ as shown in Fig. 2b), outside of which the resolution is gradually reduced. Tests with lower resolutions $(50 \mathrm{~m})$ were done to confirm that this resolution is adequate to resolve the shoaling ISWs in shallow water. Vertically, a terrainfollowing coordinate with 200 grid cells is used, and a vertically varying resolution is adopted in the deep water to improve resolution across the pycnocline. These resolutions are consistent with the sensitivity experiments of Lamb and Warn-Varnas (2015). On the ISW propagation path, stations A and B are selected to show tidal currents in shallow water (Fig. 1b, depth $80 \mathrm{~m}$ ) and deep water (Fig. 1c, depth $1500 \mathrm{~m}$ ), respectively. The barotropic tidal currents are obtained from the Oregon State University Tidal Inversion Software (OTIS7.1) (Egbert and Erofeeva 2002). The tidal ellipses indicate that the $\mathbf{M}_{2}$ tidal current is predominant in the study region, with an amplitude of $0.22 \mathrm{~m} \mathrm{~s}^{-1}$ at station A. Hence, we consider only the $\mathrm{M}_{2}$ tide in this study for simplicity. The $\mathrm{M}_{2}$ tidal current is driven at the right boundary (depth $3000 \mathrm{~m}$ ) where we specify the barotropic current as $U_{b}(t)=U_{0} \sin (\omega t+\varphi)$, with $\omega=1.4075 \times 10^{-4} \mathrm{~s}^{-1}$ being the frequency of the $\mathbf{M}_{2}$ tide. Here, the initial phase $\varphi$ is varied to make waves shoal at different phases of the barotropic tidal current, as summarized in Table 1 . The left boundary is open with a same equal volumes flux as the right boundary.

\section{c. Experiment design}

Parameters for the numerical experiments are listed in Table 1. A total of 20 shoaling ISW cases have been simulated 

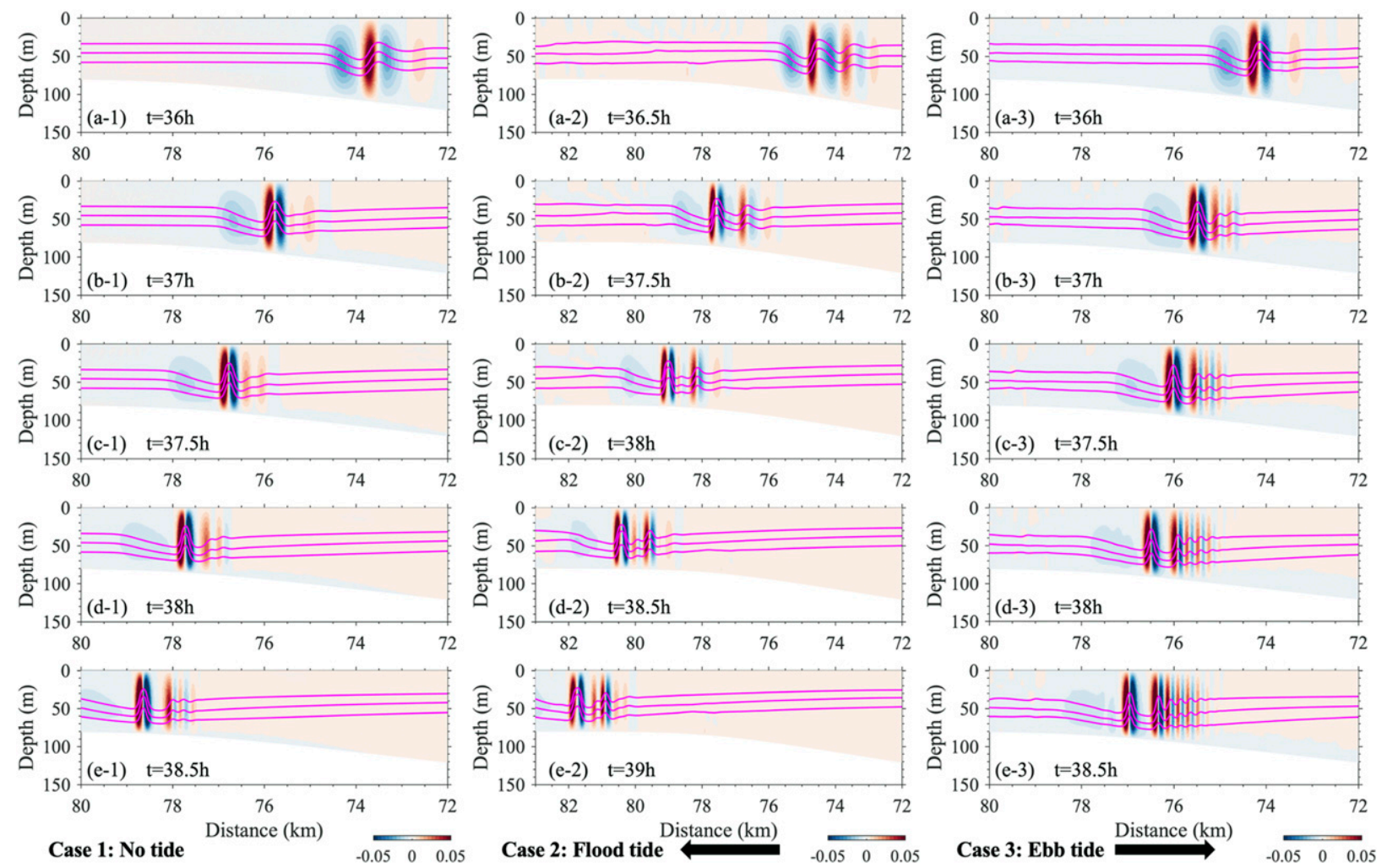

FIG. 3. Snapshots of vertical velocity (color; $\mathrm{m} \mathrm{s}^{-1}$ ) and the corresponding density field (magenta contours) in Cases 1-3 (three columns).

(a)-(e) Different times are shown, and the following numbers are the case number. The nominal critical point is at $x=77.8 \mathrm{~km}$.

and grouped into five sets. The first set consisting of Cases 1-3, based on stratification $\rho_{1}$, is employed to illustrate the influence of the tidal phase on the shoaling process as the waves pass through the critical point. In Cases 2 and 3 the shoaling ISW approaches the location of the unperturbed critical point during the flood (Case 2) and ebb (Case 3) tides, respectively, while in Case 1 there is no barotropic tidal current as in simulations of Lamb and Warn-Varnas (2015). Referring to the tidal currents over the shallow shelf from OTIS output, we use tidal currents with an amplitude of $0.3 \mathrm{~m} \mathrm{~s}^{-1}$ at $80-\mathrm{m}$ depth in most experiments. Similar to Cases 1-3, Cases 1L-3L with larger-amplitude $(45 \mathrm{~m})$ initial ISWs are carried out to compare with Cases 1-3 (ISW amplitude of $25 \mathrm{~m}$ ). An additional simulation, Case $2 \mathrm{~g}$, uses the setup of Case 2 but with $g=$ $0 \mathrm{~m} \mathrm{~s}^{-2}$, which means that there is no baroclinic current and no shoaling ISW. This case is meant to illustrate how the barotropic tidal current modifies the stratification in the absence of locally generated baroclinic tides. Combined with Case 2, Cases 4 and 5 are regarded as the second set to investigate the sensitivity of the ISW evolution to the strength of the tidal current. Cases 6-8 and Cases 9-11 are the third and fourth sets, which use stratifications $\rho_{2}$ and $\rho_{3}$, respectively, to illustrate the sensitivity of tidal modulation to stratification. The fifth set of experiments, including Case 12-16 and Case 2, is employed to examine the effects of bathymetry. The difference between Case 2 and Case 12 is used to examine the effect of the bump at $x=-12.5 \mathrm{~km}$. Cases 13-14 and Case 2 are compared to discuss the influence of the slope on tidal modulation with a bump at $x=-12.5 \mathrm{~km}$, while Case 12 and Cases $15-16$ have different upper slopes without the bump.

\section{d. Slope criticality}

Baroclinic tides are generated as barotropic tidal currents flow over sloping topography. The main features can be characterized by the slope criticality (Cacchione et al. 2002; Klymak et al. 2011)

$$
\gamma=\frac{\text { slope }}{\text { ray }}=\frac{|d h / d x|}{\left[\left(\omega^{2}-f^{2}\right) /\left(N^{2}-\omega^{2}\right)\right]^{1 / 2}},
$$

where the numerator is the topographic slope and the denominator is the slope of an internal wave beam of tidal frequency ( $N$ is the buoyancy frequency, $\omega$ the tidal frequency, and $f$ the Coriolis frequency). The slope is supercritical (subcritical) when the slope criticality is above (below) unity. Energy in incident internal waves will be reflected back into the deep water after interacting with a supercritical slope. In contrast, much of the energy is transmitted onto the continental shelf when the slope is subcritical. The slope is near-critical when the slope criticality is close to unity. Near-critical regions are often associated with elevated levels of turbulent dissipation and mixing due to enhanced wave breaking (Moum et al. 2002; Nash et al. 2004). The slope of our model bathymetry is 0.007 for $80 \mathrm{~km} \geq x \geq 0 \mathrm{~km}$ and 0.05 


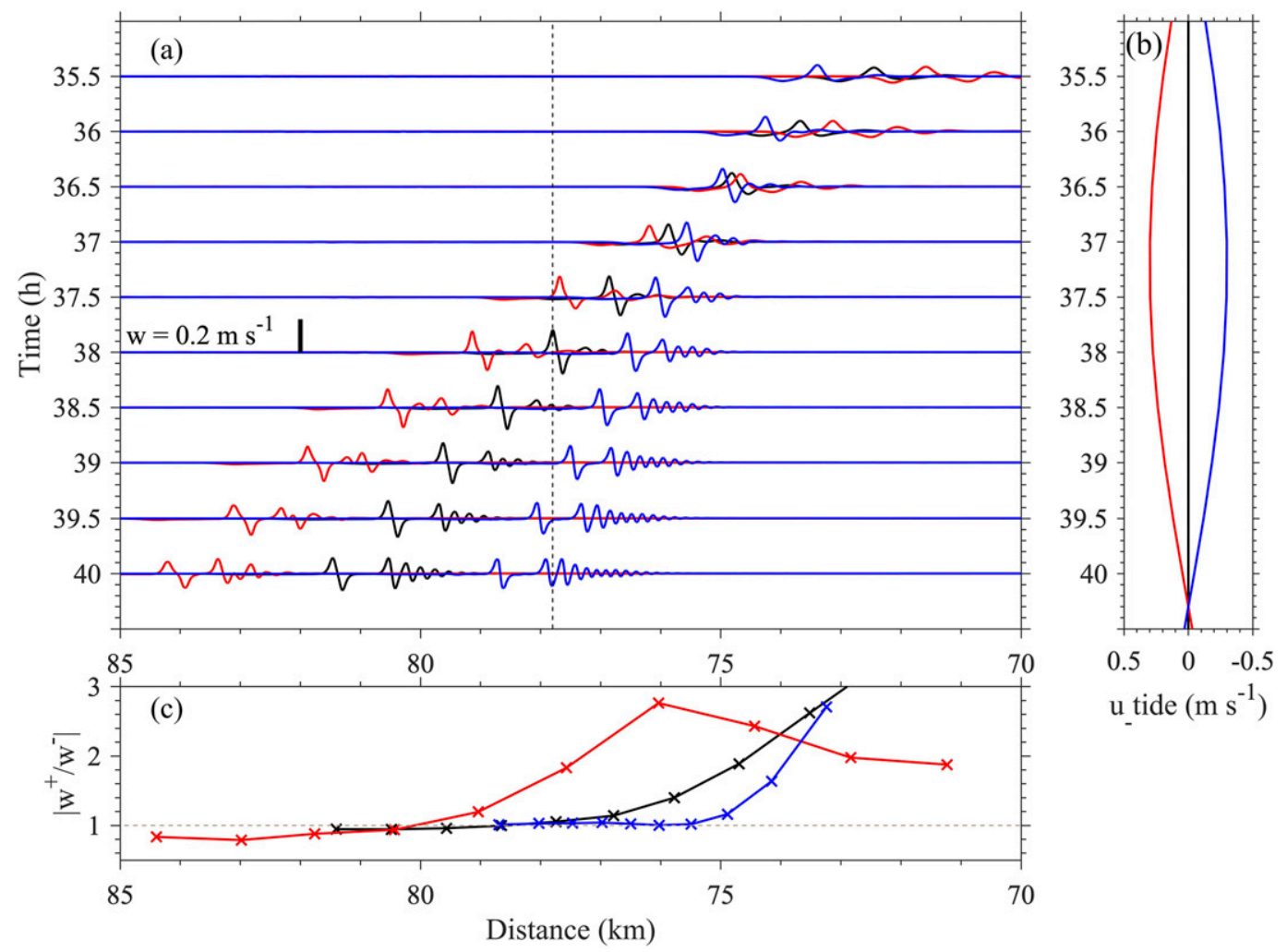

FIG. 4. (a) Variations of the vertical velocity: Case 1 (black), Case 2 (red), and Case 3 (blue). The black dashed line indicates the location of the undisturbed critical point at $x=77.8 \mathrm{~km}$. (b) Barotropic tidal currents at $x=90 \mathrm{~km}$ with positive values being shoreward. (c) Ratio of the maximum upward velocity $\left(w^{+}\right)$to the downward velocity $\left(w^{-}\right)$for the leading wave.

for $-40 \mathrm{~km} \leq x \leq-12.5 \mathrm{~km}$. Figure $2 \mathrm{c}$ indicates that the slope is supercritical near the bump at $x=-12.5 \mathrm{~km}$ and subcritical elsewhere. This suggests that baroclinic tides will be generated locally near the bump. In these experiments, most of the shoreward propagating baroclinic tidal energy will be transmitted onto the shallow shelf, where the slope at $x \geq 0 \mathrm{~km}$ is subcritical.

\section{Results and discussion}

\section{a. Effects of tides and a criterion for determining the formation of ISWs of elevation}

The deformation and evolution of shoaling ISWs can be illustrated through snapshots of vertical velocity and isopycnal contours. In Fig. 3, we compare results for Cases 1-3 to see how different phases of the tide affect the shoaling ISW: In Cases 2 and 3 , the shoaling ISW approaches the nominal critical point at $x=77.8 \mathrm{~km}$ during the flood (Case 2) and ebb (Case 3 ) tides while there is no barotropic tidal current in Case 1. Note, however, that the waves have been propagating for approximately $36 \mathrm{~h}$ from their initial locations.

Five snapshots for each case are used to highlight the evolution of the shoaling ISW as it propagates toward and through the critical point. At the earliest times [Figs. 3a(1)-3a(3)] the ISW of depression has already been deformed into the well-known asymmetric depression with a gently sloping front and steep back due to its proximity to the critical point (albeit still several kilometers away) and the rapidly changing water depth. Correspondingly, the downward current at the front of the leading depression is much weaker than the upward current at its rear. Immediately behind, a wave of elevation, or pedestal (e.g., Lamb and Warn-Varnas 2015), has started to form. As time progresses this elevation strengthens and gradually evolves into an ISW of elevation characterized by a strong upward-downward velocity pair [e.g., Figs. 3e(1)-3e(3)]. This wave trails a leading depression which determines the background stratification and currents in which the ISW of elevation forms (see Lamb and Warn-Varnas 2015). The downward velocity in the front of the leading depression weakens continuously. Furthermore, secondary waves form following the leading ISW of elevation.

Although it is difficult to distinguish when and where the ISW of elevation first forms as it is never isolated from other waves, we can do some qualitative analysis. The elevation ISW in Case 1 looks symmetric at $t=38 \mathrm{~h}$ with the formation of trailing new waves. The wave location in Fig. 3d(1) is close to the nominal critical point ( $x=77.8 \mathrm{~km})$. As well, we can see the symmetric structure along with generation of trailing new waves near $x=81.0 \mathrm{~km}$ in Case 2 [Fig. $3 \mathrm{~d}(2)$ ] and at $x=$ $75.5 \mathrm{~km}$ in Case 3 [Fig. 3b(3)].

To get a quantitative understanding of when ISWs of elevation form, we plot the variations of the extreme value of the vertical velocity over the water depth as a function of $x$ at 

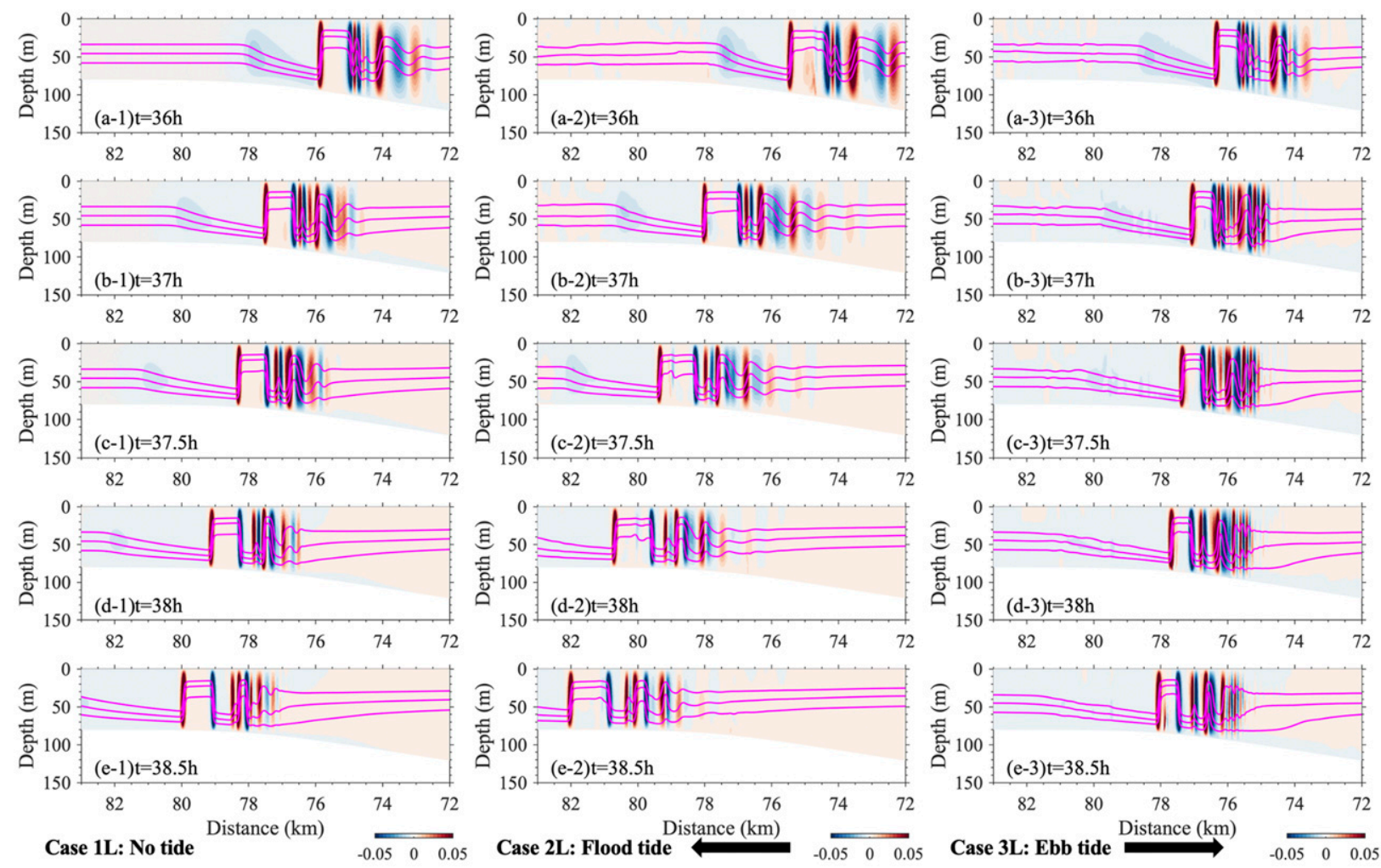

FIG. 5. As in Fig. 3, but for larger-amplitude shoaling ISWs: (left) Case 1L, (center) Case 2L, and (right) Case 3L.

several times for Cases 1-3 in Fig. 4a. Elevation ISWs are characterized by an upward-downward current pair. In Fig. 4a, the strong upward current at the rear of the leading depression and its following downward current, which strengthens with time, clearly indicates the formation and evolution of an elevation wave. Correspondingly, the ratio of the maximum upward velocity $\left(w^{+}\right)$to the maximum downward velocity $\left(w^{-}\right)$illustrates a decreasing trend as the elevation wave develops. In Fig. 4c, the ratio is plotted as a function of the front of the wave which is taken as the horizontal location of maximum vertical velocity. In Case 1 the ratio decreases from greater than 3 to be equal to 1 at $x=77.9 \mathrm{~km}$, which is close to the nominal critical point $\alpha=0 \mathrm{~s}^{-1}$ for the undisturbed stratification. Therefore, here we put forward another criterion to determine the location of the critical point, that is, using the midpoint of the maximum upward and downward velocities in the leading wave meeting the condition of

$$
\left|w^{+} / w^{-}\right|=1 \text {. }
$$

Applying this criterion to the other two cases, we see the location of $\left|w^{+} / w^{-}\right|=1$ is at $x=80.3 \mathrm{~km}$ in Case 2 and at $x=$ $75.6 \mathrm{~km}$ in Case 3. This criterion works well in these cases although there is no guarantee that the value will always decrease to 1 . As a result, in addition to modifying the propagation speed, tidal currents affect the location where ISWs of elevation first appear. The critical point is modulated to move onshore under the effect of the flood tide but offshore during the ebb tide.

Since the amplitude of ISWs in the deep SCS is often larger than $100 \mathrm{~m}$ (Huang et al. 2016), we carried out another group of experiments with larger-amplitude ISWs (Fig. 5). Although the initial wave amplitude in these cases is $45 \mathrm{~m}$, still smaller than the largest amplitude of ISWs in the SCS, these largeamplitude ISWs deforms into long waves with flat-centered crests. Such a wave structure does not generally occur on the continental shelf of the SCS (e.g., Liu et al. 2004; Zhao et al. 2004; Bai et al. 2019) but similar wave forms have been observed in field measurements (e.g., Fig. 6 in Ramp et al. 2004) and in numerical simulations (Lamb and Warn-Varnas 2015). Strong breaking and overturning occur in the wave tail (not visible at this scale; see Lamb and Warn-Varnas 2015). Hence, we will focus on results obtained with waves of initial amplitude $25 \mathrm{~m}$ to investigate the effects of tidal currents on shoaling ISWs.

It is more difficult to directly see when ISWs of elevation first appear for the larger-amplitude cases, but the ratio criterion, i.e., $\left|w^{+} / w^{-}\right|=1$, works well in such complex conditions. We apply the ratio criterion and compare the Case $1 \mathrm{~L}$ with Case 1 in Fig. 6. The distance between the maximum upward and downward velocities is $0.94 \mathrm{~km}$ in Case $1 \mathrm{~L}$, much larger than the distance of $0.16 \mathrm{~km}$ in Case 1 . Owing to the stronger current induced by the larger-amplitude ISW, $\left|w^{+} / w^{-}\right|$is initially larger in Case 1L. However, the ratio decreases rapidly and is close to 1 at a similar location with Case 1 . According to the criterion 


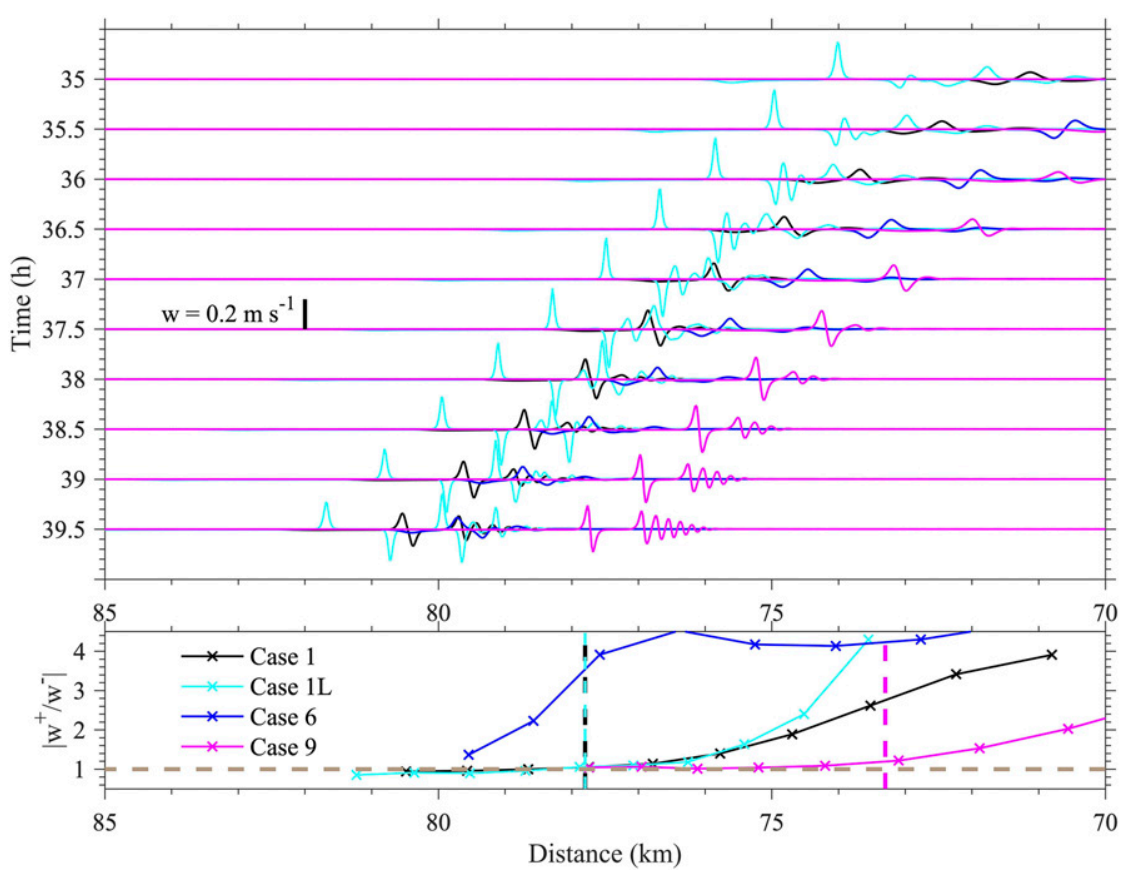

FIG. 6. As in Fig. 4, but for comparing the four cases without tidal currents: Case 1 (black), Case 1L (cyan), Case 6 (blue), and Case 9 (magenta). Dashed lines indicate the critical point location predicted by $\alpha=0 \mathrm{~s}^{-1}$.

using $\alpha=0 \mathrm{~s}^{-1}$, the critical points in Case 1 and Case $1 \mathrm{~L}$ are at the same location because these cases use the same stratification and neither include background currents. Similar predictions from the two methods also indicate the applicability of the ratio criterion. Moreover, the location of $\left|w^{+} / w^{-}\right|=1$ is close to the nominal critical point predicted using $\alpha=0 \mathrm{~s}^{-1}$, although the value of $\left|w^{+} / w^{-}\right|$has been very close to 1 at $76.8 \mathrm{~km}$ in the two cases. The results also suggest that the $\alpha=0 \mathrm{~s}^{-1}$ criterion is feasible for analyzing the variations of ISW transformation modulated by tidal currents.

\section{b. Time-varying critical point}

The basic features and process of tidal modulation on shoaling ISWs have been illustrated by comparing Cases 1-3. Here we investigate the dynamical mechanism of this phenomenon, based on the contributions of tidally driven fluctuations in stratification and sheared currents to ISW polarity variations as predicted by the value of $\alpha$ evaluated using Eq. (1). In the absence of background currents, $\alpha$ is a function of $x$ only as shown in Fig. 2d. However, as the stratification and baroclinic currents are modulated by tidal currents, so are the eigenfunction $\phi$ and the phase speed $c$. As a result, $\alpha$ becomes a function of both space and time. We focus on the variation of the most offshore critical point in this study as this is the critical point first encountered by shoaling ISWs.

Similarly, a pseudo-critical point is calculated to compare with the above value based on Eq. (1) by using the time-varying stratification with a depth-independent current, i.e., $U(x, z, t)=$ $U_{b}(x, t)$. We call this the pseudocritical point because shear is not considered. The depth-independent current does not affect the value of $\alpha$. As a result, the contribution of sheared currents to the location of the critical point can be qualitatively tested by comparing the difference between the locations of the critical point and the pseudocritical point.

The spatial-temporal variations of ISW polarity conversion modulated by tidal currents can be seen in Fig. 7. Corresponding to the tidal currents in Cases 1-3 (Fig. 7a), the time-varying locations of the critical point and the pseudocritical point are shown in Fig. 7b, and the time series of isopycnal displacement at the nominal critical point $(x=77.8 \mathrm{~km})$ is illustrated in Fig. 7c. For Case 1, with no tidal current, $x_{\alpha=0}$ is constant in time. By contrast, $x_{\alpha=0}$ moves onshore from 77.8 to $90 \mathrm{~km}$ during the flood tide and offshore to $74.8 \mathrm{~km}$ during the ebb tide. The isopycnal at the nominal critical point is elevated (depressed) during the flood (ebb) tide indicated by positive (negative) isopycnal displacement $\delta z$ in Fig. 7c. In Figs. 7b and $7 \mathrm{c}$, the differences between the effects of the flood and ebb tides are caused by larger tidal excursions in shallower water. Moreover, the critical point movement changes its direction (offshore or onshore) when the isopycnal displacement $\delta z$ changes sign (negative or positive). The time series of $x_{\alpha=0}$ has a better correlation with $\delta z$ than it does with barotropic tidal currents, suggesting that the cross-shelf movement of the critical point modulated by tidal currents is mainly determined by stratification variations. This is further corroborated by comparing the locations of the pseudocritical and critical points. In Fig. 7b, the locations are similar most of the time, suggesting that the time-varying stratification plays a more important role in the tidal modulation of ISW polarity conversion. Large differences in the locations occur during the first 

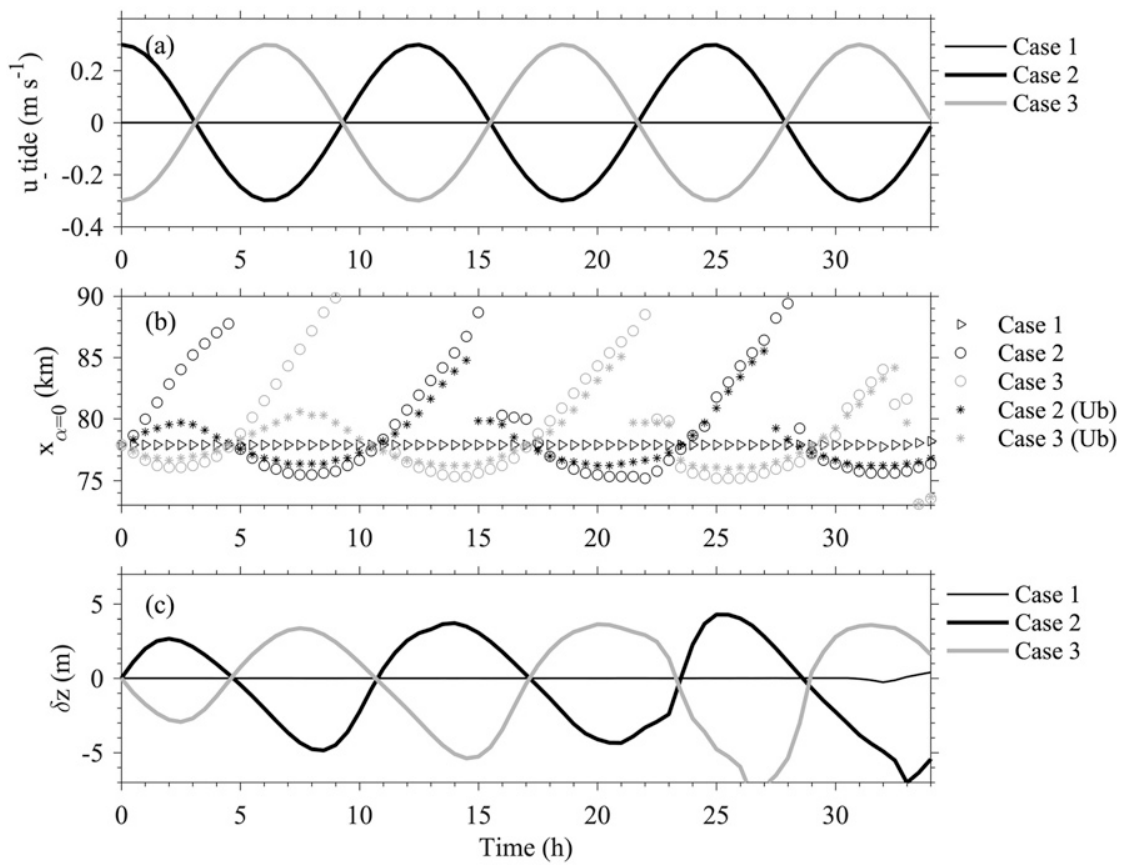

FIG. 7. (a) Barotropic tidal currents over the continental shelf (at $x=90 \mathrm{~km}$ ). (b) Location of the deepest critical point as a function of time calculated using the simulated current outputs (Cases 1-3) and location of the pseudocritical point calculated using only the barotropic current, i.e., Cases 2 (Ub) and Cases 3 (Ub). (c) Vertical displacement of isopycnal $\sigma_{\theta}=23.6 \mathrm{~kg} \mathrm{~m}^{-3}$ (undisturbed depth $45.5 \mathrm{~m}$ ) at the location $x=77.8 \mathrm{~km}$ in Cases $1-3$.

tidal period and at times when the tidal current changes direction from onshore to offshore. It is a period when locally generated baroclinic tides change the background sheared currents, resulting in distinct differences between the locations of the pseudocritical point and the critical point. Therefore, the effects of baroclinic currents cannot be ignored in calculating $\alpha$.

Details of the critical point movement are shown in the snapshots of the spatial distribution of $\alpha$ in Fig. 9. At $t=0$, the distribution of $\alpha$ is the same in all cases. For Case 1 which has no background currents, $\alpha$ is invariant with time. In Cases 2 and 3 , the value of $\alpha$ around the nominal critical point decreases (increases) during the flood (ebb) tide. Correspondingly, the most offshore critical point moves onshore (offshore). This explains the movement of $x_{\alpha=0}$ shown in Fig. 7b. During some time periods, there is more than one critical point, e.g., Case 2 at $t=6 \mathrm{~h}$ (Fig. 8c) and Case 3 at $t=12 \mathrm{~h}$ (Fig. 8e). After their appearance over the shallow shelf, these critical points move with the propagating baroclinic tides. We focus on the most offshore critical point in this study, although this result suggests that shoaling ISWs, which propagate faster than the baroclinic tide, may encounter a second critical point.

\section{c. Role of barotropic and baroclinic currents on critical point variation}

Figure 7 indicates the importance of stratification variations on the cross-shelf movement of the critical point, as well as the importance of the baroclinic currents. Meanwhile, one can see that the isopycnal variation is phase-locked with the tidal forcing. We now consider the role of the locally generated baroclinic currents in more detail. To illustrate the contribution of barotropic currents to the time varying stratification, we conduct another simulation, Case $2 \mathrm{~g}$, by setting gravity to zero in Case 2 so that there is no baroclinic current or shoaling ISW. As shown in Fig. 9b, the variation of the location of the critical point based on the stratification in Case $2 \mathrm{~g}$ is periodic, varying between 75.8 and $80.1 \mathrm{~km}$, which is consistent with the $\delta z$ variations in Fig. 9c. Additionally, we can see that the barotropic tide drives the isopycnal up (down) when flooding (ebbing). The isopycnal goes back to its initial depth at the end of a tidal period. These results not only emphasize the importance of stratification on critical point movement, but also indicate the potential contribution of barotropic current to the modulation of ISW polarity conversion.

The isopycnal fluctuations at different locations are compared in Cases $2 \mathrm{~g}$ and 2 in Figs. 8d-8e: three sites on the slope at $x=40,60$, and $77.8 \mathrm{~km}$, and one site on the shelf at $x=$ $90 \mathrm{~km}$. Under the effect of the barotropic tide, the isopycnal fluctuation is largest at $x=77.8 \mathrm{~km}$, and decreases with increasing distance away from the critical point. In Case $2 \mathrm{~g}$, the isopycnal does not fluctuate at $x=90 \mathrm{~km}$ because the bottom is flat and hence there is no vertical motion. In contrast, in Case 2 this isopycnal at $x=90 \mathrm{~km}$ starts to vary after $t=4 \mathrm{~h}$ when the locally generated baroclinic tides arrive. The amplitudes of the isopycnal fluctuations in Case 2 are comparable at the four locations after the first tidal period, because of the generation and propagation of baroclinic tides. The propagation time of baroclinic energy results in the phase difference at different locations. 


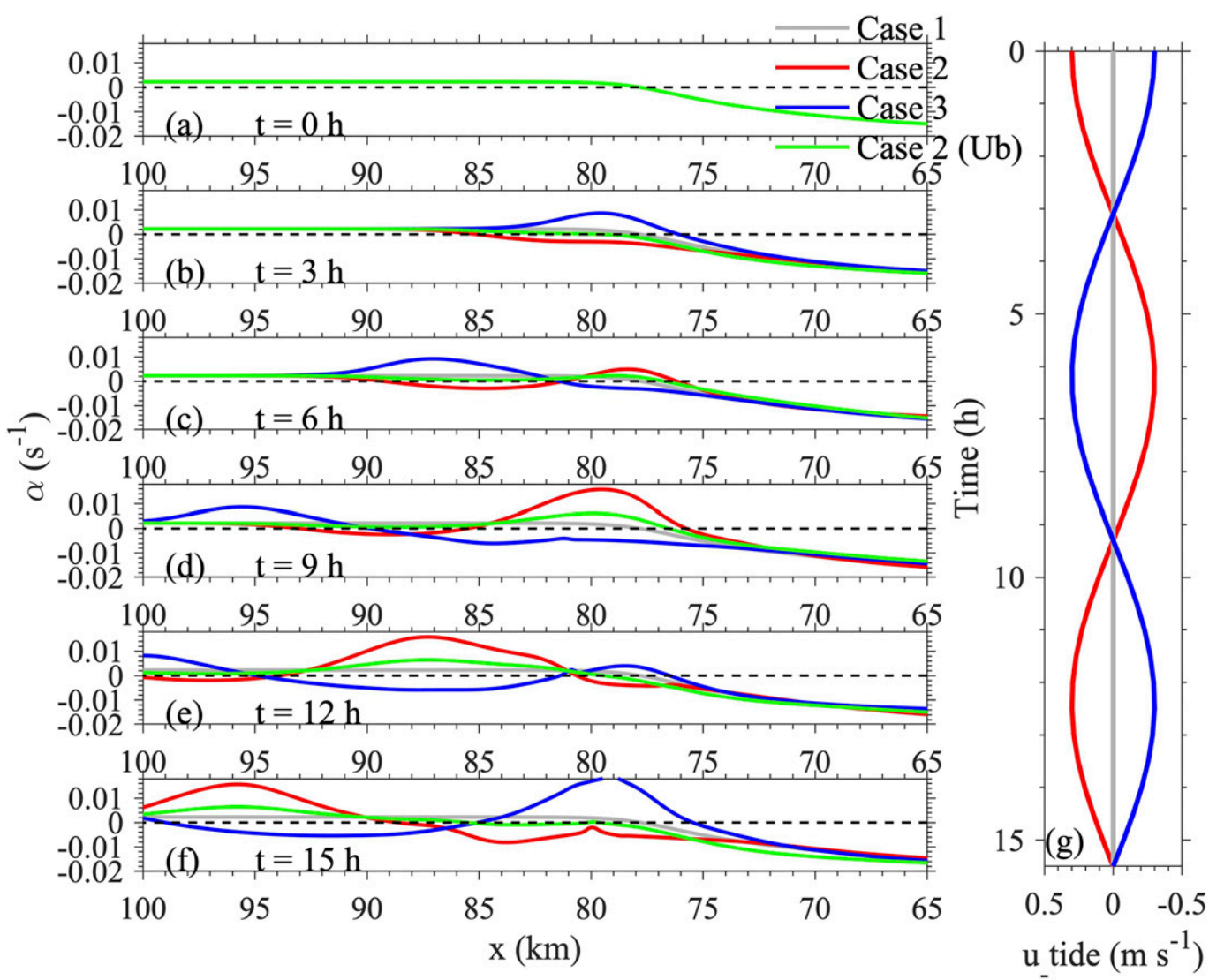

FIG. 8. (a)-(f) Spatial distribution of the quadratic nonlinear coefficient $\alpha$ at different times for Cases 1-3. The green line indicates the calculation based on Case $2(\mathrm{Ub})$. (g) The corresponding barotropic tidal currents over the continental shelf (at $x=90 \mathrm{~km}$ ). Note direction of positive current in $(\mathrm{g})$.

\section{d. Sensitivity of critical point variation to tidal current amplitude}

In the above experiments, the shallow water tidal current amplitude of $0.3 \mathrm{~m} \mathrm{~s}^{-1}$ was set to ensure that the $\mathrm{M}_{2}$ tidal current over the continental shelf is similar to that predicted by OTIS. Here, we carry out another set of experiments to investigate the sensitivity of critical point variation to the tidal current amplitude, comparing Case 2 with Case 4 (stronger current: $0.45 \mathrm{~m} \mathrm{~s}^{-1}$ at $80-\mathrm{m} \mathrm{depth}$ ) and Case 5 (weaker current: $0.19 \mathrm{~m} \mathrm{~s}^{-1}$ at $80-\mathrm{m}$ depth). As shown in Fig. 10, the increasing tidal current does induce a larger isopycnal displacement $\delta z$, with its value being in direct proportion to the tidal current amplitude. As a result, the distance of the critical point movement is largest in Case 4 and smallest in Case 5 as expected, indicating that the tidal current amplitude is also a primary factor. To make a comparison of sensitivity experiments, we carry out other investigations on critical point variation based on tidal current amplitude of $0.3 \mathrm{~m} \mathrm{~s}^{-1}$ in the following sections.

\section{e. Sensitivity of critical point variation to stratification}

We now compare results obtained using the three stratification profiles shown in Fig. 2a which primarily differ in their depth of maximum buoyancy frequency. Cases $6-8$ for the shallower pycnocline stratification profile $\rho_{2}$ and Cases
9-11 for the deeper pycnocline stratification profile $\rho_{3}$ are similar to Cases 1-3. The same analyses are done for the two new sets of experiments as well as the base experiments Cases 1-3.

Figure 11 shows the snapshots of shoaling ISWs in Case 1, Case 6, and Case 9 for which there are no tidal currents. Times of the snapshots are different in the three cases, owing to different propagation speeds for the three stratifications. Without tidal forcing in the three cases, the location of the critical point is only determined by the stratification. One can see that the ISW of depression has evolved into a depression followed by an ISW of elevation at $t=38 \mathrm{~h}$ near $x=78 \mathrm{~km}$ in Fig. 11a for Case 1. This transformation in Case 9 (Fig. 11c) occurs much earlier $(t=35 \mathrm{~h})$ and at a deeper site $(x=74 \mathrm{~km})$ because the critical point is in deeper water $\left(x_{\alpha=0}=77.8 \mathrm{~km}\right.$ in Case 1 , $x_{\alpha=0}=73.3 \mathrm{~km}$ in Case 2). In contrast, in Case 6 (Fig. 11b), for which there is no critical point, the ISW fissions into several waves of depression. There is also a trailing packet of ISWs between 70 and $75 \mathrm{~km}$ which is generated as the shoaling ISW passes over the bump at $x=12.5 \mathrm{~km}$. The predictions based on $\alpha=0$ and $\left|w^{+} / w^{-}\right|=1$ for Case 6 and Case 9 are also shown in Fig. 6. For the shallower pycnocline $\rho_{2}, \alpha>$ $0 \mathrm{~s}^{-1}$ and $\left|w^{+} / w^{-}\right|>1$ in the undisturbed fluid. For the deeper pycnocline $\rho_{3}, \alpha=0$ and $\left|w^{+} / w^{-}\right|=1$ occur at an 


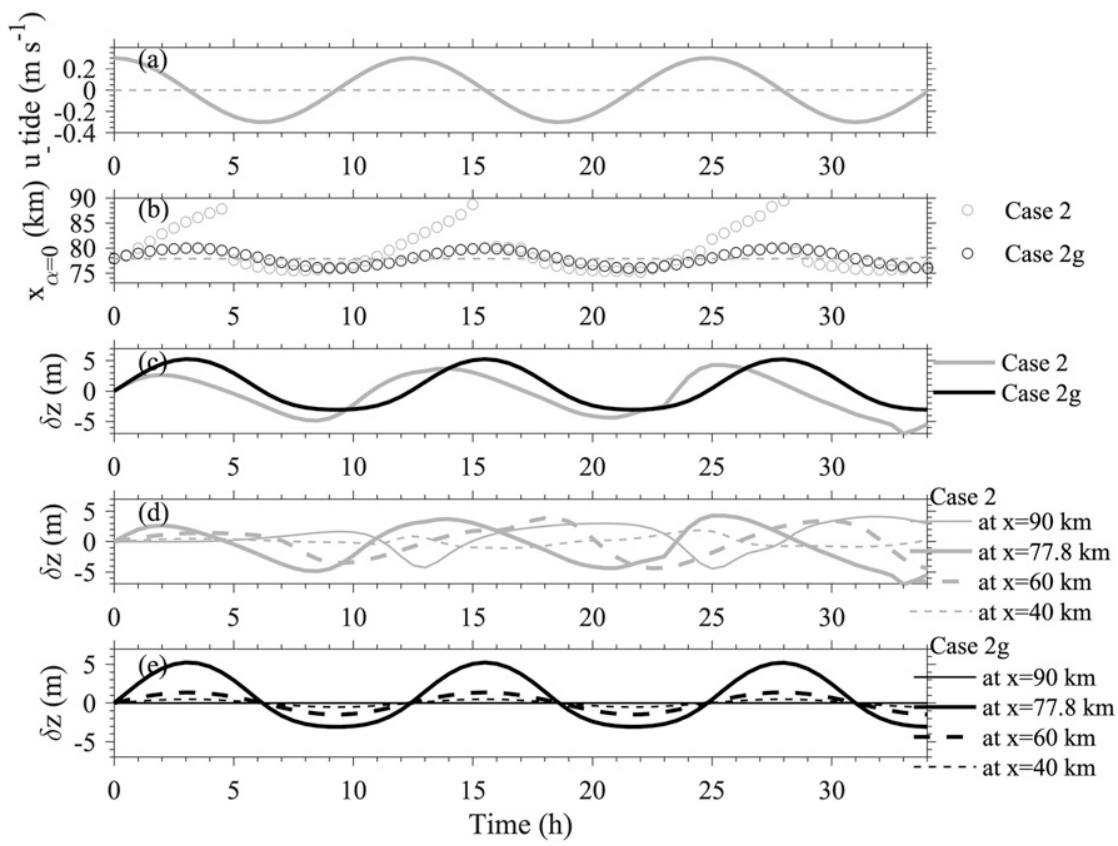

FIG. 9. (a)-(c) As in Figs. 7a-7c, but for Case 2 and Case 2g: (a) the barotropic tidal currents at $x=90 \mathrm{~km}$, (b) location of the critical point as a function of time, and (c) vertical displacement of isopycnal $\sigma_{\theta}=23.6 \mathrm{~kg} \mathrm{~m}^{-3}$. Variations of the isopycnal displacement at different locations $(x=40,60$, and 77.8, and $90 \mathrm{~km}$ ) are indicated for (d) Case 2 and (e) Case $2 \mathrm{~g}$.

offshore location. And the location $x_{\alpha=0}$ is $2 \mathrm{~km}$ offshore of the location of $\left|w^{+} / w^{-}\right|=1$, suggesting that it takes time for the elevation ISW to become symmetric when it forms at the back of the leading depression.
The absence of critical points for the shallower pycnocline $\rho_{2}$ can be changed by tidal currents as isopycnals rise and fall during the flood and ebb tides (Fig. 12c). The isopycnal displacement is up to $4.0 \pm 1.0 \mathrm{~m}$ in Cases 7 and 8 . In Fig. 12b, the
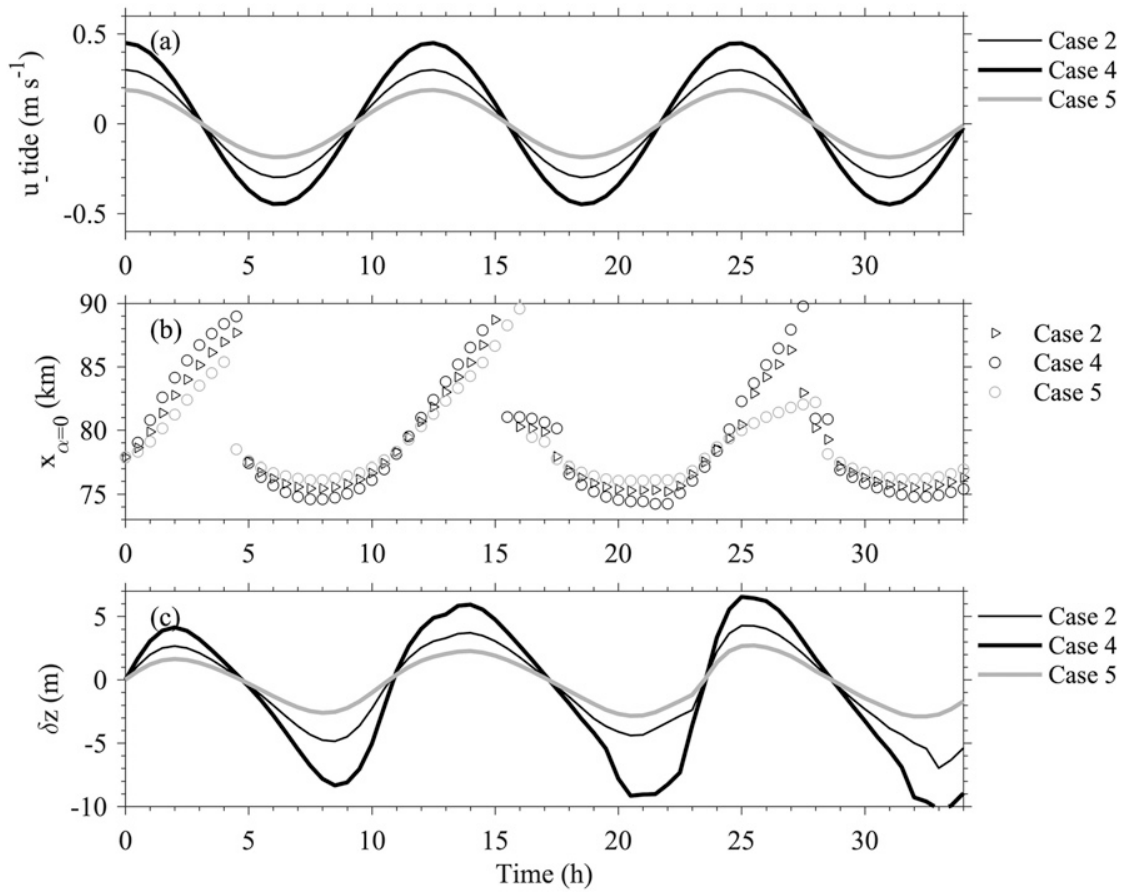

FIG. 10. As in Fig. 7, but for Cases 2, 4, and 5 using different tidal current amplitude. 


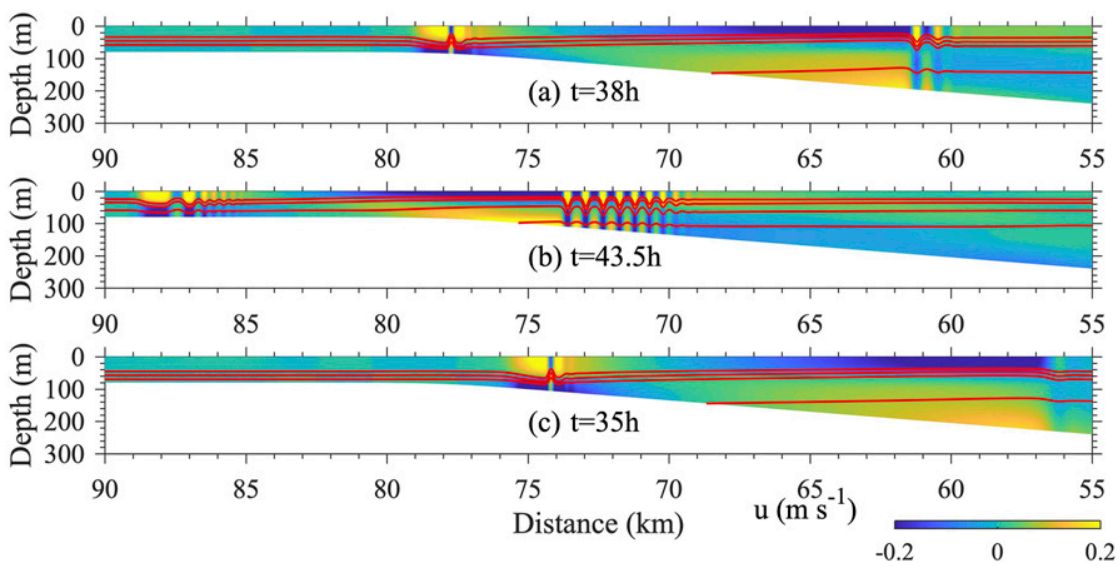

FIG. 11. Snapshots of baroclinic velocity (color) and the corresponding density field (contours) for different stratifications with now tidal currents: (a) Case 1 (stratification $\rho_{1}$ ) at $t=$ $38 \mathrm{~h}$, (b) Case 6 (stratification $\rho_{2}$ ) at $t=43.5 \mathrm{~h}$, and (c) Case 9 (stratification $\rho_{3}$ ) at $t=35 \mathrm{~h}$.

critical point first shows up at $t=7.5 \mathrm{~h}$ in Case 7 and at $t=13.5 \mathrm{~h}$ in Case 8 , when the isopycnal is depressed by $\delta z=-3.5 \mathrm{~m}$ in the two cases. Although Case 8 is initialized at peak ebb tide, its maximum isopycnal displacement is only $2.3 \mathrm{~m}$ so a critical point does not appear during the first isopycnal depression. The stratification profile $\rho_{2}$ has its maximum buoyancy frequency at 30-m depth and a critical point can be generated in a water depth of $85.0 \mathrm{~m}$ when there are tidal currents. On the one hand, we can conclude that the generation of a critical point can be largely attributed to the tidally modulated stratification, because of the phase-locked variation of isopycnal variation (Fig. 12c) with the tidal forcing (Fig. 12a). On the other hand, we cannot neglect the contribution of sheared currents in this set of experiments. By comparing Case 7 with Case 7 (Ub) and Case 8 with Case 8 (Ub) in Fig. 12b, one can see that critical points appear, but there are no pseudocritical points, hence the baroclinic sheared currents are essential to the creation of transitory critical points.

The above analysis is in accordance with the spatialtemporal variations of $\alpha$ as shown in Fig. 13. Tidal modulation of the distribution of $\alpha$ shows similar feature to those seen
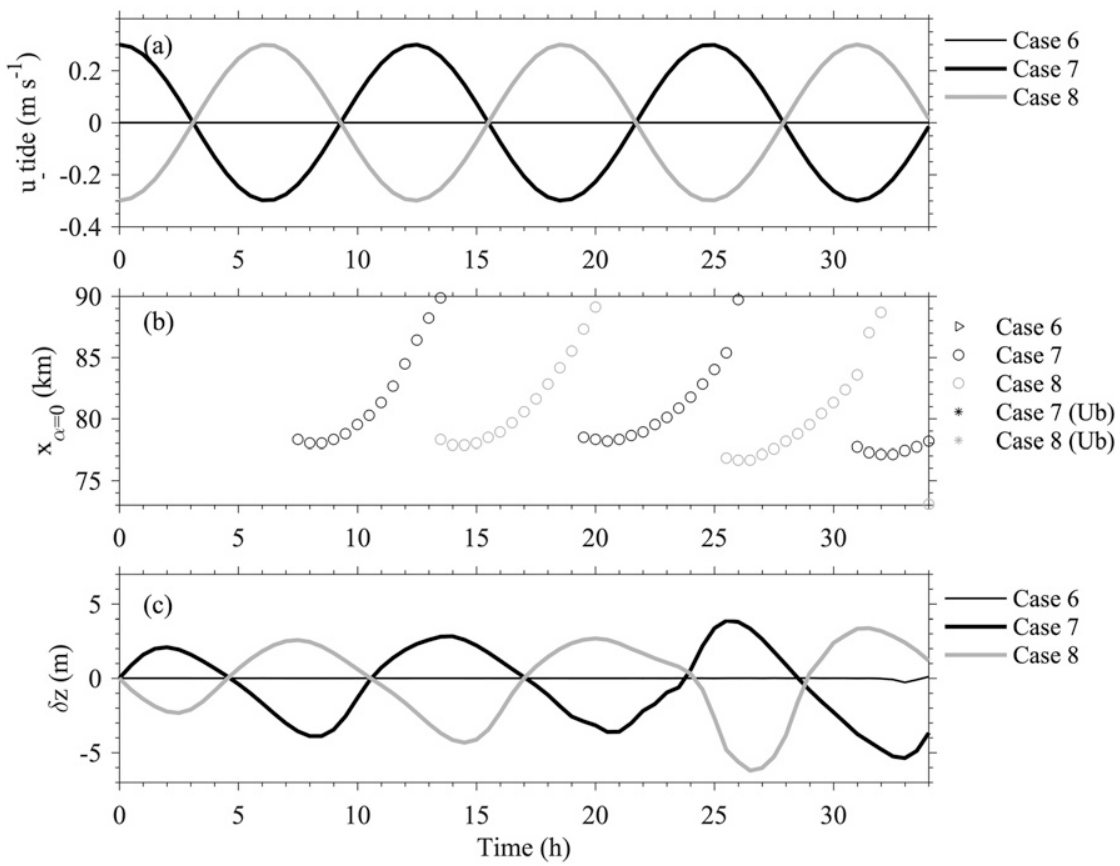

FIG. 12. As in Fig. 7, but for Cases 6, 7, and 8 based on stratification profile $\rho_{2}$ (shallower pycnocline). Note that results of Case 6 , Case 7 (Ub), and Case 8 (Ub) do not appear in (b) because there is never a critical point in these cases. 


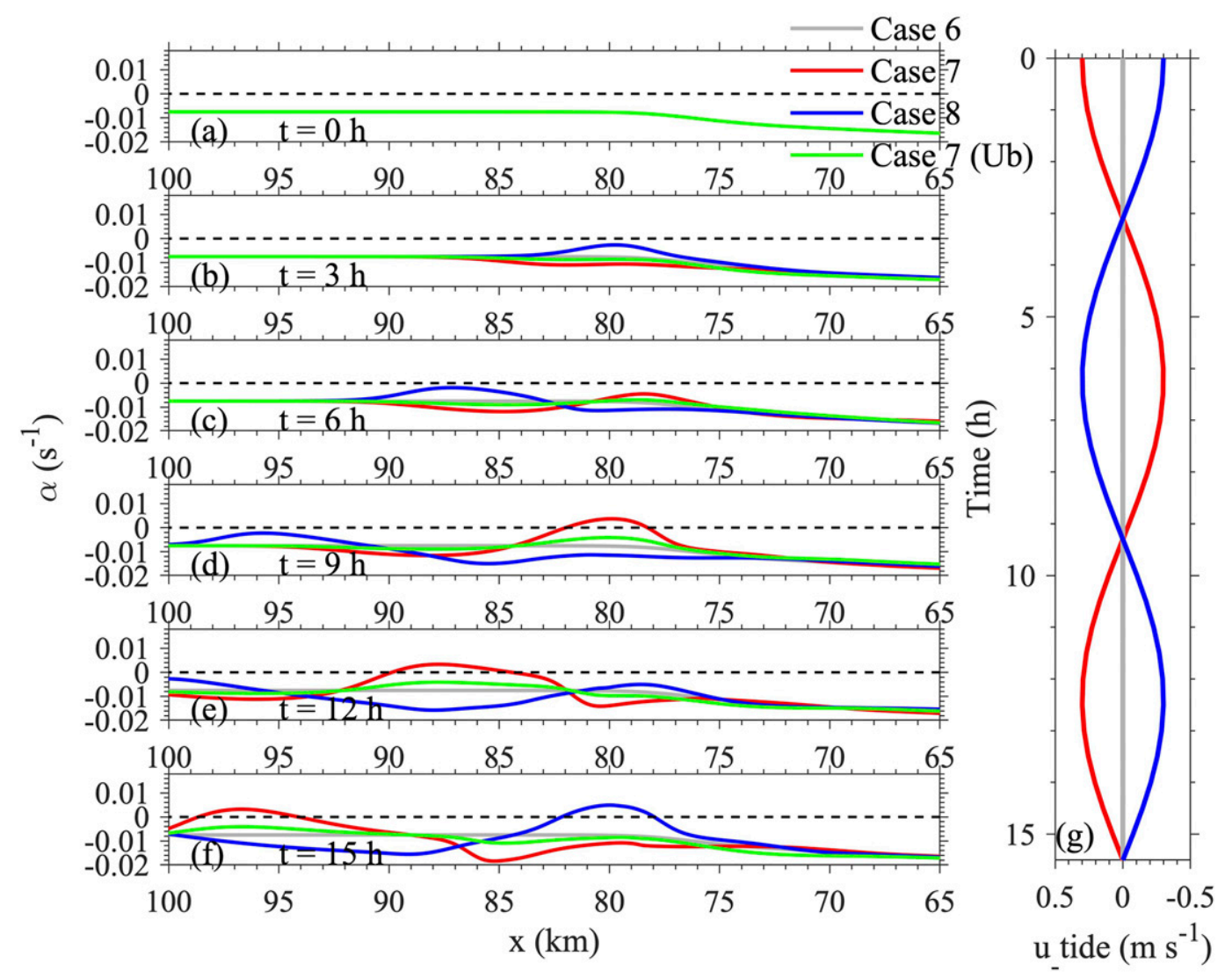

FIG. 13. As in Fig. 8, but for Cases 6, 7, and 8 based on stratification profile $\rho_{2}$.

in Fig. 8 for Cases 1-3. In Fig. 12, critical points appear near the peak of the ebb tide, e.g., at $t=7.5 \mathrm{~h}$ in Case 7 and $t=13.5 \mathrm{~h}$ in Case 8. Although no critical point shows up during the first ebb tide period in Case 8 (Fig. 12b), one can see the maximum $\alpha$ is very close to zero in Figs. 13b-13e. Such a quasi-vanishing $\alpha$ would affect the amplitude and strongly modify the evolution of shoaling ISWs.

In contrast to Case 6 with the shallowest pycnocline, the deeper pycnocline for $\rho_{3}$ ensures that there always exists a critical point in Cases 9-11. In Fig. 14, the nominal critical point is located at $x=73.3 \mathrm{~km}$ (depth $111.9 \mathrm{~m}$ ) in Case 9. The critical point moves shorter distances in Cases 10-11 than it does in Cases 2-3 because of the weaker tidal currents in the deeper water. In Fig. 14b, the maximum offshore displacements of the critical point are 1.5 and $1.2 \mathrm{~km}$ in Cases 10 and 11 , respectively. The onshore displacements are less than $0.5 \mathrm{~km}$ in both cases. It seems that the critical point is locked on the continental slope. This is because isopycnal variations are not large enough to change the polarity of ISWs in other regions as shown in Fig. 15. In deeper water, the tidal currents are a bit weaker, and $\alpha$ near the nominal critical point does not vary as much as it does on the shelf or shelf break. On the other hand, one can see that the decrease of $\alpha$ during the flood tide is still able to generate new critical points, for instance in Case 10 at $t=15 \mathrm{~h}$ (Fig. 15f) and Case 11 at $t=9 \mathrm{~h}$ (Fig. 15d). The newly generated critical points can move further onshore over the shelf and may influence the propagating ISWs on the shelf.

\section{f. Sensitivity of critical point variation to bathymetry}

The tidal modulation of the location of the critical point depends on the bathymetric slope, which affects the generation of baroclinic tidal currents. In this section we investigate the influence of bathymetry, mainly focusing on the role of the bump at $x=-12.5 \mathrm{~km}$ in Fig. $2 \mathrm{~b}$ and the upper slope connecting it to the shallow shelf. The bump is considered because of its high slope criticality and its influence on internal wave generation (Xie et al. 2019) and because it contributes to fission of the shoaling ISW. Hence, five more experiments, Cases 1216 which use the same stratification and tidal forcing as Case 2, are carried out. Their bathymetries, slope criticality for the $\mathrm{M}_{2}$ tide and distribution of $\alpha$ are shown in Fig. 16 and detailed in Table 1.

The bump's effect on the critical point movement is investigated by comparing Case 2 (with the bump) and Case 12 (without the bump). The removal of the bump in Case 12 makes the bathymetry in Case 12 subcritical (Fig. 16b). It is close to critical near $x=-20.0 \mathrm{~km}$. Comparing the critical point variations in Fig. $17 \mathrm{~b}$ and isopycnal displacement in Fig. 17c, one can see that the differences between Cases 2 and 12 show up at $t=25 \mathrm{~h}$. The distance between the locations $x=-20.0 \mathrm{~km}$ (the near-critical slope) and $x=77.8 \mathrm{~km}$ (the 

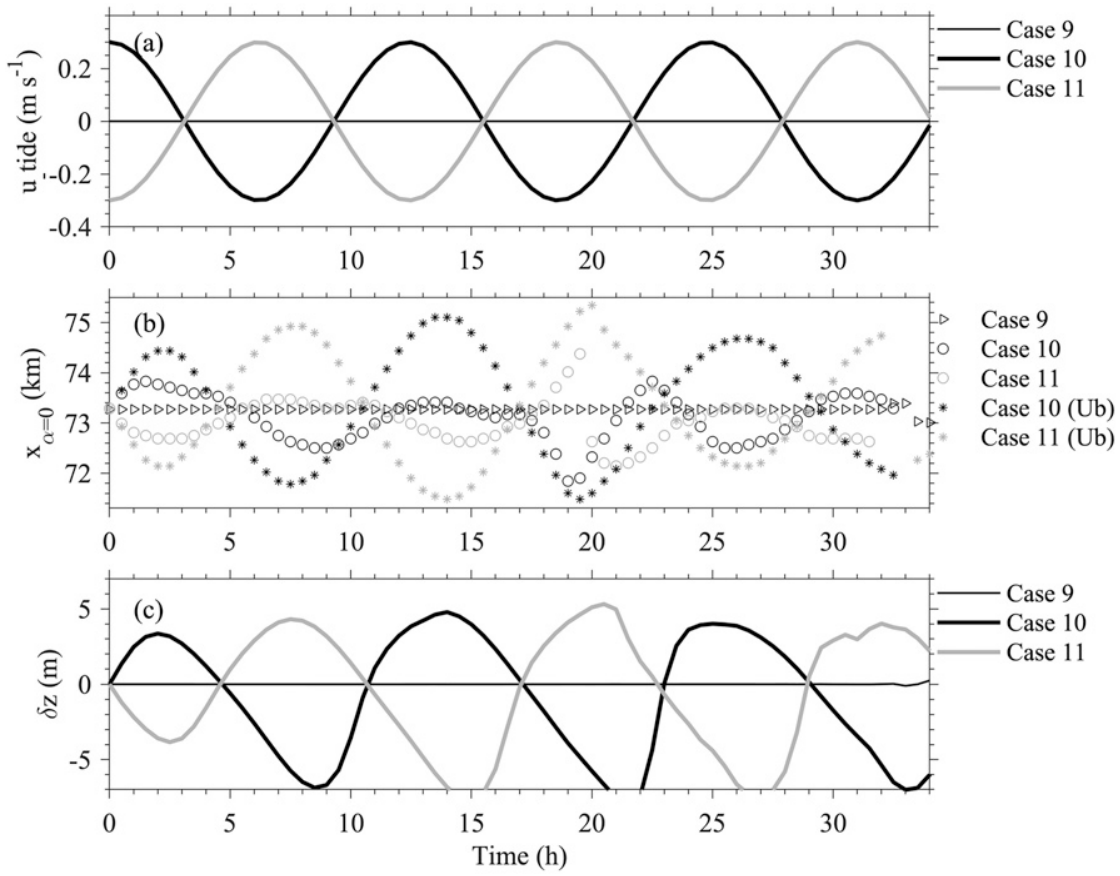

FIG. 14. As in Fig. 7, but for Cases 9, 10, and 11 based on stratification profile $\rho_{3}$ (deeper pycnocline). It is noted that the ordinate scale in (b) is different from that in Fig. $7 \mathrm{~b}$.

nominal critical point) is $97.8 \mathrm{~km}$, which agrees with the travel distance of mode- 1 internal waves in $25 \mathrm{~h}$. The result indicates that baroclinic tides generated over the underwater bump affect the isopycnal variations and critical point movement after a certain time. It also suggests that the presence of remote sources of baroclinic tides will also affect the location of the critical point thereby reducing the predictability of features of internal waves in the real ocean
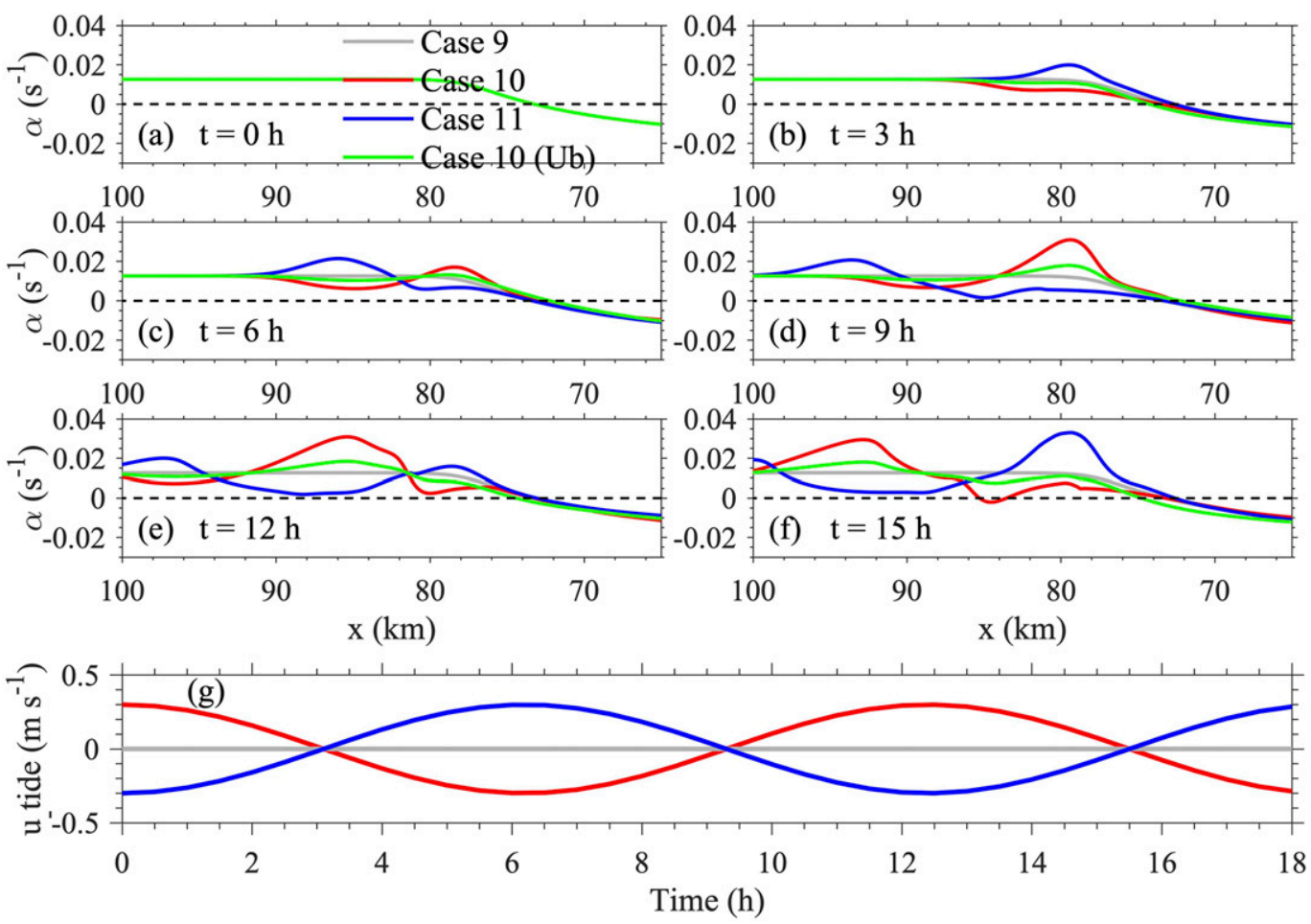

FIG. 15. As in Fig. 8, but for Cases 9, 10, and 11 based on stratification profile $\rho_{3}$. 

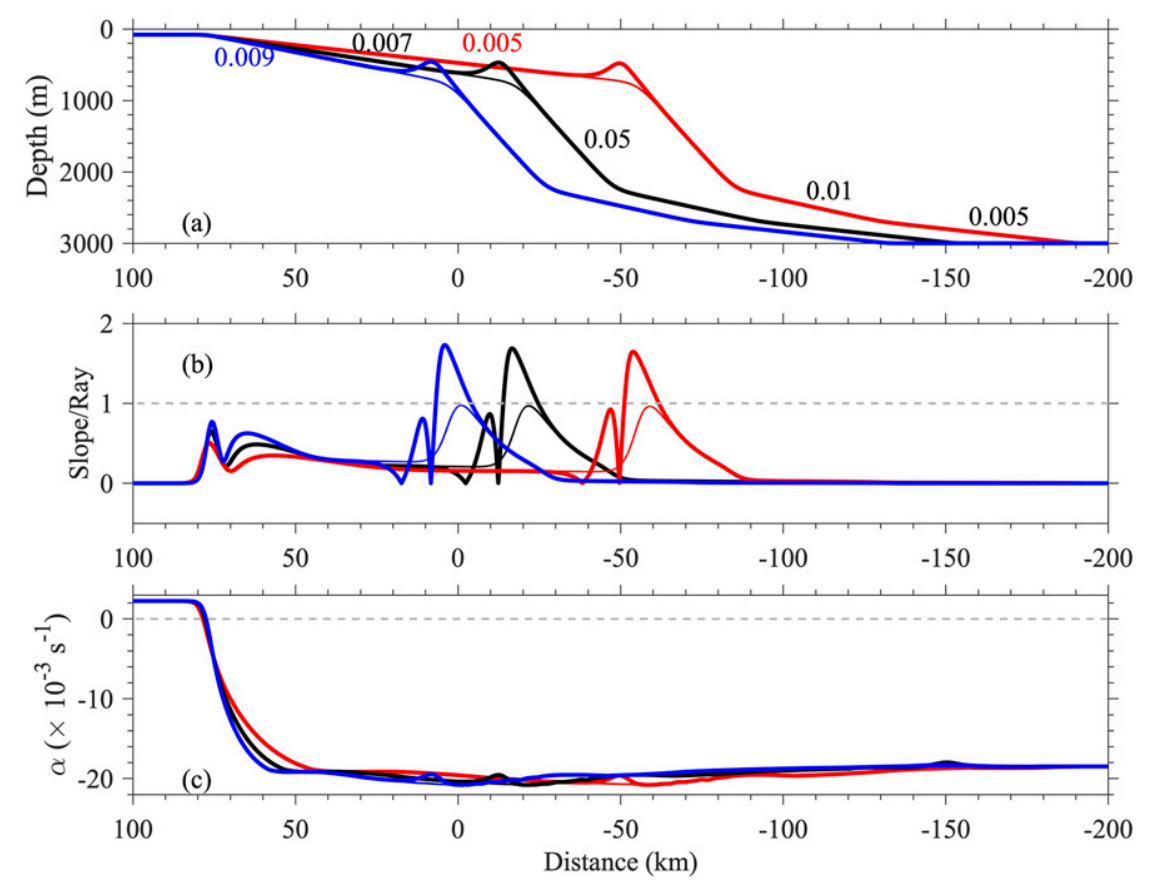

FIG. 16. As in Figs. 2b-d: (a) bathymetry for Case 12 (thin black), Case 13 (thick red), Case 14 (thick blue), Case 15 (thin red), Case 16 (thin blue) and Case 2 (thick black), with the numbers showing the slopes. The corresponding slope criticality for the $\mathrm{M}_{2}$ tide for the six cases in (b), and quadratic nonlinear coefficient $\alpha$ in (c).

as concluded by Nash et al. (2012) and investigated by Gong et al. (2019).

The slope's effect on critical point movement is examined by comparing Cases 2 and 13-14 (with the bump), as well as by comparing Case 12 and 15-16 (without the bump) in Fig. 18. Relative to the bathymetry of Case 2 (Case 12), the slope to the left of the bump is decreased to 0.005 in Case 13 (Case 15) and increased to 0.009 in Case 14 (Case 16), respectively. The location of
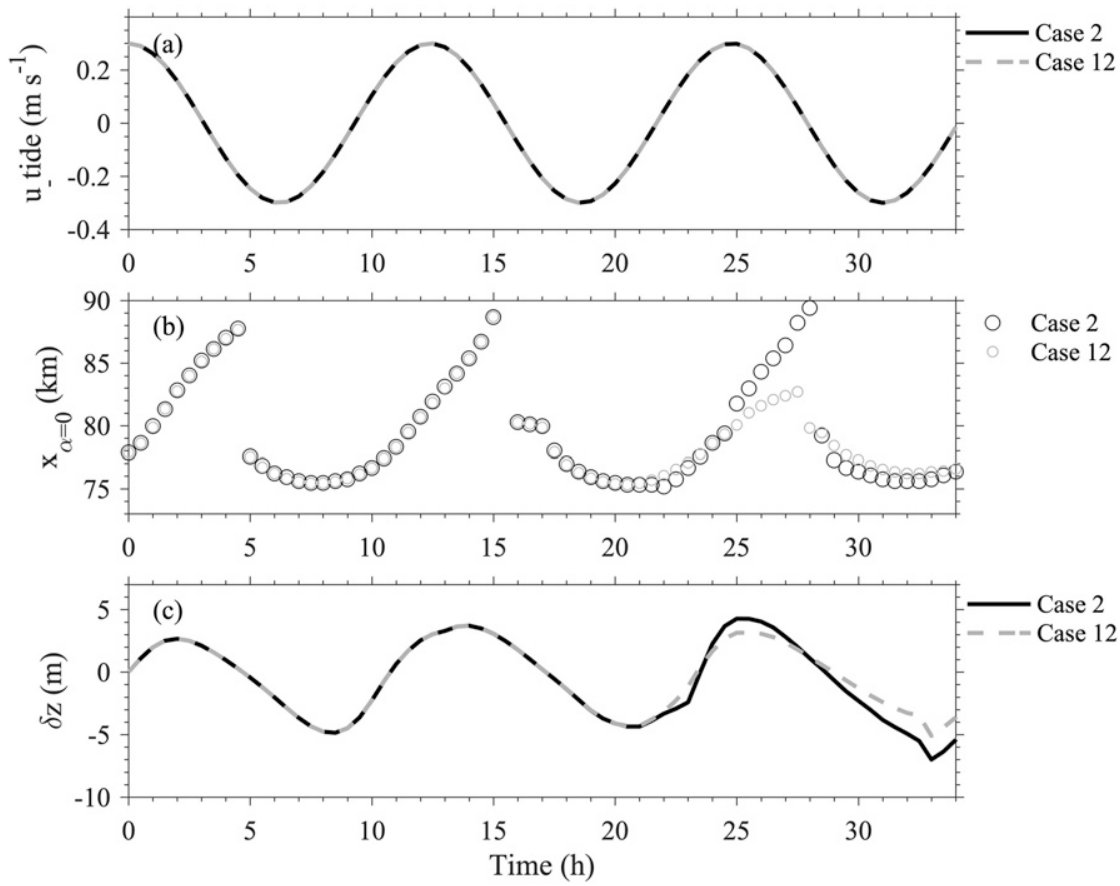

FIG. 17. As in Fig. 7, but for comparing Case 2 and Case 12. 

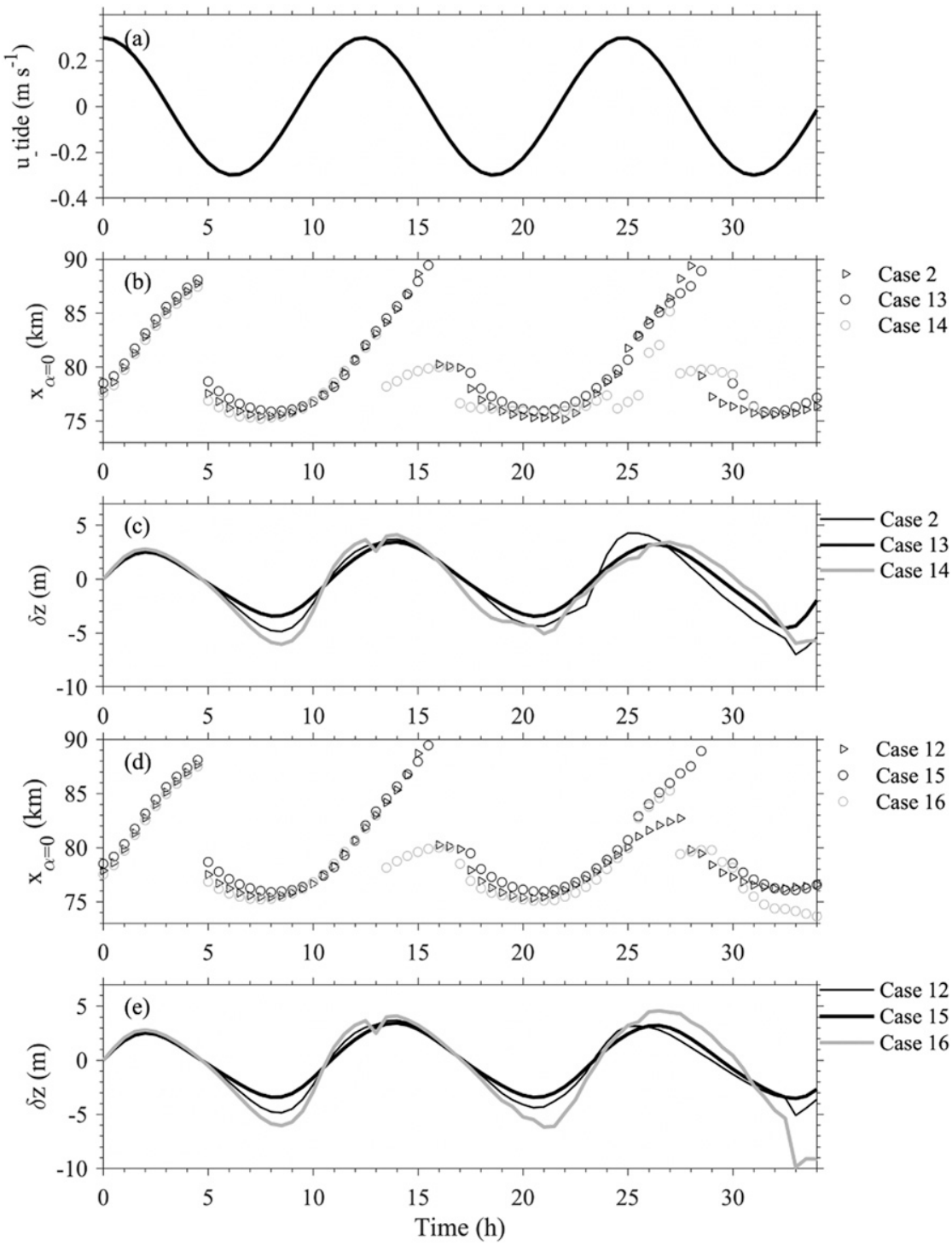

FIG. 18. As in Fig. 7, but for comparing the effects of slope based on Case 2 (general slope of 0.007 in black triangle and line), Case 13 (gentle slope of 0.005 in black circle and line), and Case 14 (steep slope of 0.009 in gray circle and line) with the underwater bump in (b) and (c), while comparing the effects of slope based on Case 12 (general slope), Case 15 (gentle slope), and Case 16 (steep slope) without the bump in (d) and (e).

the underwater bump and the lower steep slope varies as shown in Fig. 16a, because the upper slope near the shelf break is modified while preserving the location of the shelf break. The nominal critical points are close for the three slopes as indicated in Fig. 16c. For the cases with the bump (Cases 2 and 13-14 in Figs. 18b,c), the isopycnal displacement is similar for the three slopes during the flood tide but different during the ebb tide. The steeper slope results in a larger isopycnal displacement (Fig. 18c), but the difference in isopycnal displacement is not enough to significantly change $\alpha$, so that the difference of the critical point movement in the three cases is less than $1 \mathrm{~km}$. The critical point displacement shows similar variations in all the three cases, which, however, is broken due to the effects of baroclinic tidal currents generated from the underwater bump and their varying arrival time, for example at $t=25 \mathrm{~h}$ for Case 2 and earlier (later) for Case 14 (Case 13). The difference of $x_{\alpha=0}$ in Case 14 relative to the other two cases between 13.5 and $15.5 \mathrm{~h}$ is because of the generation of a new critical point. Similar results can be seen in Figs. $18 \mathrm{~d}$ and $18 \mathrm{e}$ for the cases without the bump. Because of the reduced influence of baroclinic waves generated by the bump, the deviation of $x_{\alpha=0}$ in Fig. 18d is much less than that in Fig. 18b.

\section{Conclusions, implications, and limitations}

The polarity of an ISW is determined by the stratification and background current shear. Given its shear-free nature, the 
barotropic tidal current has commonly been thought to have negligible influence on the location of the critical point. In this study, we have carried out numerical simulations using a fully nonlinear numerical model to shed light on the effects of tidal currents on the spatial-temporal variations of the location of the critical point and the resulting transformation of a shoaling ISW. Introducing barotropic tidal currents, the pycnocline depth is significantly modulated by cross-shelf tidal currents. The barotropic tidal currents drive isopycnals up and down on the slope, but as this occurs, baroclinic tides are generated locally, radiating energy away, further modulating the stratification and introducing sheared currents. The isopycnal and baroclinic current variations are phase-locked to the tidal currents. When a shoaling ISW experiences flood tide while propagating close to the critical point, the tidal current not only increases the propagation speed of the shoaling ISW, but also shifts the critical point onshore, delaying the transformation of the shoaling ISW. In contrast, ebb current decreases the wave speed, and results in an earlier and further offshore transformation.

In our study, isopycnal variation induced by tidal currents is the key factor for the tidal modulation of the location of the critical point. The variations of stratification are affected by both the barotropic tides and the baroclinic tides generated by tide-topography interactions. Background sheared currents associated with baroclinic tides are important as well, especially when the stratification cannot meet the generation condition of critical point, for example when the pycnocline depth is less than half of the total water depth. We also investigated the sensitivity of critical point variation to the slope of the bathymetry. The results indicate that steeper bathymetry may induce larger isopycnal variations and bigger cross-shelf movement of the critical point.

This study highlights the spatial-temporal variation of ISW transformation modulated by tidal currents. Tidal currents extend the region where the ISW transformation occurs. Nutrient transport and ocean mixing are affected by ISW transformation because of enhanced mixing (e.g., Klymak and Moum 2003; Orr and Mignerey 2003; Shroyer et al. 2009) and because of reversals of waveform and wave-induced velocity in the resulting ISWs of elevation (e.g., Orr and Mignerey 2003). In addition to fissioning into a wave train with shorter wavelengths (e.g., Bai et al. 2019), bottom drag and cross-shelf transport of fluid and biology would be enhanced by waves of elevation in the near shore (e.g., Klymak and Moum 2003; Scotti and Pineda 2004), and tidal currents change the depth dependence of these processes. As ISWs and tidal currents are ubiquitous features in coastal regions of the world's oceans, this process, i.e., the tidal modulation of shoaling ISWs, is of global importance. Compared to the influence of seasonally varying stratification and mesoscale eddies (monthly time scales), the modulation of tidal currents is more frequent and occurs on much shorter time scales. This study provides an example in the SCS, where ISWs are among the most energetic in the world's oceans, but the effects of tidal currents we see in our simulations should have quite generic implications.

In this study, the bottom slope is mainly subcritical based on the bathymetric features in the ASIAEX region. The effects of steeper bathymetry, e.g., a near-critical and supercritical slope, is of interest and should be investigated in future work. The real ocean is more complex because of the presence of both local and remote sources of baroclinic tides, making the nature of internal waves at times unpredictable on continental shelves (Nash et al. 2012; Gong et al. 2019). The influence of remotely generated baroclinic tides on shoaling ISWs also warrants investigation in the near future, as well as the effects of threedimensional bathymetry resulting in spatially varying tidal currents and wave refraction, and of cross-shelf variations in the stratification. Although the way it is advected back and forth by the tides would not be changed, a horizontally varying stratification would change the location of the critical point and would also introduce an additional mechanism by which the shoaling wave evolves as it shoals. Refraction of tidal currents over three-dimensional bathymetry would alter the propagation of ISWs as well as the location of critical points. Therefore, to fully understand the transformation of shoaling waves and the resulting implications for mixing, transport, and productivity in the SCS and elsewhere, a three-dimensional model with realistic wave amplitudes and background currents is needed.

Acknowledgments. This work was supported by the National Natural Science Foundation of China (91858201, 91958203, 42006019, and 41721005), as well as grants from the Natural Sciences and Engineering Research Council of Canada (NSERC) and the Canadian Foundation for Innovation. X. Bai also acknowledges International Postdoctoral Exchange Fellowship Program (2018) by China Postdoctoral Council and China Scholarship Council (201506310091). Information of the barotropic tides is obtained from OTIS (http://volkov.oce.orst.edu/ tides/). The simulations were conducted at the facilities of Shared Hierarchical Academic Research Computing Network (SHARCNET: www.sharcnet.ca) and Compute/Calcul Canada.

\section{REFERENCES}

Aghsaee, P., L. Boegman, and K. G. Lamb, 2010: Breaking of shoaling internal solitary waves. J. Fluid Mech., 659, 289-317, https://doi.org/10.1017/S002211201000248X.

Alford, M. H., and Coauthors, 2015: The formation and fate of internal waves in the South China Sea. Nature, 521, 65-69, https://doi.org/10.1038/nature14399.

Bai, X., X. Li, K. G. Lamb, and J. Hu, 2017: Internal solitary wave reflection near Dongsha Atoll, the South China Sea. J. Geophys. Res. Oceans, 122, 7978-7991, https://doi.org/ 10.1002/2017JC012880.

_- Z. Liu, Q. Zheng, J. Hu, K. G. Lamb, and S. Cai, 2019: Fission of shoaling internal waves on the northeastern shelf of the South China Sea. J. Geophys. Res. Oceans, 124, 4529-4545, https://doi.org/10.1029/2018JC014437.

Bell, J. B., and D. L. Marcus, 1992: A second-order projection method for variable-density flows. J. Comput. Phys., 101, 334348, https://doi.org/10.1016/0021-9991(92)90011-M.

Cacchione, D. A., L. F. Pratson, and A. S. Ogston, 2002: The shape of continental slopes by internal tides. Science, 296, 724-727, https://doi.org/10.1126/science.1069803.

Cai, S., J. Xie, J. Xu, D. Wang, Z. Chen, X. Deng, and X. Long, 2014: Monthly variation of some parameters about internal 
solitary waves in the South China Sea. Deep-Sea Res. I, 84, 7385, https://doi.org/10.1016/j.dsr.2013.10.008.

Egbert, G. D., and S. Y. Erofeeva, 2002: Efficient inverse modeling of barotropic ocean tides. J. Atmos. Oceanic Technol., 19, 183-204, https://doi.org/10.1175/1520-0426(2002) 019<0183:EIMOBO > 2.0.CO;2.

Fan, Z., X. Shi, A. K. Liu, H. Liu, and P. Li, 2013: Effects of tidal currents on nonlinear internal solitary waves in the South China Sea. J. Ocean Univ. China, 12, 13-22, https://doi.org/ 10.1007/s11802-013-1870-0.

Fu, K.-H., Y.-H. Wang, L. St. Laurent, H. Simmons, and D.-P. Wang, 2012: Shoaling of large-amplitude nonlinear internal waves at Dongsha Atoll in the northern South China Sea. Cont. Shelf Res., 37, 1-7, https://doi.org/ 10.1016/j.csr.2012.01.010.

Gargettt, A. E., and B. A. Hughes, 1972: On the interaction of surface and internal waves. J. Fluid Mech., 52, 179-191, https:// doi.org/10.1017/S0022112072003027.

Gong, Y., M. D. Rayson, N. L. Jones, and G. N. Ivey, 2019: The effects of remote internal tides on continental slope internal tide generation. J. Phys. Oceanogr., 49, 1651-1668, https:// doi.org/10.1175/JPO-D-18-0180.1.

Grimshaw, R., E. Pelinovsky, T. Talipova, and A. Kurkin, 2004: Simulation of the transformation of internal solitary waves on oceanic shelves. J. Phys. Oceanogr., 34, 2774-2791, https:// doi.org/10.1175/JPO2652.1.

$\ldots,-\ldots, \ldots$, and O. Kurkina, 2010: Internal solitary waves: Propagation, deformation and disintegration. Nonlinear Processes Geophys., 17, 633-649, https://doi.org/10.5194/ npg-17-633-2010.

—, K. Helfrich, and A. Scotti, 2011: Large amplitude internal waves in the coastal ocean. Nonlinear Processes Geophys., 18, 653-655, https://doi.org/10.5194/npg-18-653-2011.

_ C. Wang, and L. Li, 2016: Modelling of polarity change in a nonlinear internal wave train in Laoshan Bay. J. Phys. Oceanogr., 46, 965-974, https://doi.org/10.1175/JPO-D-150136.1.

Helfrich, K. R., and R. H. Grimshaw, 2008: Nonlinear disintegration of the internal tide. J. Phys. Oceanogr., 38, 686-701, https://doi.org/10.1175/2007JPO3826.1.

Huang, X., Z. Chen, W. Zhao, Z. Zhang, C. Zhou, Q. Yang, and J. Tian, 2016: An extreme internal solitary wave event observed in the northern South China Sea. Sci. Rep., 6, 30 041, https://doi.org/10.1038/srep30041.

Jackson, C., and J. Apel, 2004: An Atlas of Internal Solitary-Like Waves and Their Properties. Global Ocean Associates, 560 pp.

Klymak, J. M., and J. N. Moum, 2003: Internal solitary waves of elevation advancing on a shoaling shelf. Geophys. Res. Lett., 30, 2045, https://doi.org/10.1029/2003GL017706.

—, R. Pinkel, C.-T. Liu, A. K. Liu, and L. David, 2006: Prototypical solitons in the South China Sea. Geophys. Res. Lett., 33, L11607, https://doi.org/10.1029/2006GL025932.

— M. H. Alford, R. Pinkel, R.-C. Lien, Y. J. Yang, and T.-Y. Tang, 2011: The breaking and scattering of the internal tide on a continental slope. J. Phys. Oceanogr., 41, 926-945, https:// doi.org/10.1175/2010JPO4500.1.

Kurkina, O. E., T. G. Talipova, T. Soomere, A. A. Kurkin, and A. V. Rybin, 2017: The impact of seasonal changes in stratification on the dynamics of internal waves in the Sea of Okhotsk. Est. J. Earth Sci., 66, 238-255, https://doi.org/ 10.3176/earth.2017.20.

Lamb, K. G., 1994: Numerical experiments of internal wave generation by strong tidal flow across a finite amplitude bank edge. J. Geophys. Res., 99, 843-864, https://doi.org/10.1029/ 93JC02514.

, 2002: A numerical investigation of solitary internal waves with trapped cores formed via shoaling. J. Fluid Mech., 451, 109-144, https://doi.org/10.1017/S002211200100636X.

—_, 2004: Nonlinear interaction among internal wave beams generated by tidal flow over supercritical topography. Geophys. Res. Lett., 31, L09313, https://doi.org/10.1029/ 2003 GL019393.

, 2007: Energy and pseudoenergy flux in the internal wave field generated by tidal flow over topography. Cont. Shelf Res., 27, 1208-1232, https://doi.org/10.1016/j.csr.2007.01.020.

— shelf: Comparisons of weakly-nonlinear and fully nonlinear models for hyperbolic-tangent stratifications. Ocean Modell., 78, 17-34, https://doi.org/10.1016/j.ocemod.2014.02.005.

_ , and A. Warn-Varnas, 2015: Two-dimensional numerical simulations of shoaling internal solitary waves at the ASIAEX site in the South China Sea. Nonlinear Processes Geophys., 22, 289-312, https://doi.org/10.5194/npg-22-289-2015.

_ , and M. Dunphy, 2018: Internal wave generation by tidal flow over a two-dimensional ridge: Energy flux asymmetries induced by a steady surface trapped current. J. Fluid Mech., 836, 192-221, https://doi.org/10.1017/jfm.2017.800.

Li, L., C. Wang, and R. Grimshaw, 2015: Observations of internal wave polarity conversion generated by a rising tide. Geophys. Res. Lett., 42, 4007-4013, https://doi.org/10.1002/ 2015GL063870.

Liao, G., X. Xu, C. Liang, C. Dong, B. Zhou, T. Ding, W. Huang, and D. Xu, 2014: Analysis of kinematic parameters of internal solitary waves in the northern South China Sea. Deep-Sea Res. I, 94, 159-172, https://doi.org/10.1016/j.dsr.2014.10.002.

Lien, R.-C., F. Henyey, B. Ma, and Y. J. Yang, 2014: Largeamplitude internal solitary waves observed in the northern South China Sea: Properties and energetics. J. Phys. Oceanogr., 44, 1095-1115, https://doi.org/10.1175/JPO-D-13-088.1.

Liu, A. K., Y. S. Chang, M.-K. Hsu, and N. K. Liang, 1998: Evolution of nonlinear internal waves in the East and South China Seas. J. Geophys. Res., 103, 7995-8008, https://doi.org/ 10.1029/97JC01918.

—, S. R. Ramp, Y. Zhao, and T. Y. Tang, 2004: A case study of internal solitary wave propagation during ASIAEX 2001. IEEE J. Oceanic Eng., 29, 1144-1156, https://doi.org/10.1109/ JOE.2004.841392.

Margolin, L. G., W. J. Rider, and F. F. Grinstein, 2006: Modeling turbulent flow with implicit LES. J. Turbul., 7, N15, https:// doi.org/10.1080/14685240500331595.

Moum, J. N., D. R. Caldwell, J. D. Nash, and G. D. Gunderson, 2002: Observations of boundary mixing over the continental slope. J. Phys. Oceanogr., 32, 2113-2130, https://doi.org/ 10.1175/1520-0485(2002)032<2113:OOBMOT >2.0.CO;2.

Nash, J. D., E. Kunze, J. Toole, and R. Schmitt, 2004: Internal tide reflection and turbulent mixing on the continental slope. J. Phys. Oceanogr., 34, 1117-1134, https://doi.org/10.1175/ 1520-0485(2004)034<1117:ITRATM >2.0.CO;2.

_ S. M. Kelly, E. L. Shroyer, J. N. Moum, and T. F. Duda, 2012: The unpredictable nature of internal tides on the continental shelves. J. Phys. Oceanogr., 42, 1981-2000, https://doi.org/ 10.1175/JPO-D-12-028.1.

Orr, M. H., and P. C. Mignerey, 2003: Nonlinear internal waves in the South China Sea: Observation of the conversion of depression internal waves to elevation internal waves. J. Geophys. Res., 108, 3064, https://doi.org/10.1029/2001JC001163. 
Osborne, A. R., and T. L. Burch, 1980: Internal solitons in the Andaman Sea. Science, 208, 451-460, https://doi.org/10.1126/ science.208.4443.451.

Ramp, S. R., and Coauthors, 2004: Internal solitons in the northeastern South China Sea, Part I: Sources and deep water propagation. IEEE J. Oceanic Eng., 29, 1157-1181, https:// doi.org/10.1109/JOE.2004.840839.

_ , J.-H. Park, Y. J. Yang, F. L. Bahr, and C. Jeon, 2019: Latitudinal structure of solitons in the South China Sea. J. Phys. Oceanogr., 49, 1747-1767, https://doi.org/10.1175/JPO-D-18-0071.1.

Sandstrom, H., and J. A. Elliott, 1984: Internal tide and solitons on the Scotian Shelf: A nutrient pump at work. J. Geophys. Res., 89, 6415-6426, https://doi.org/10.1029/JC089iC04p06415.

Scotti, A., and J. Pineda, 2004: Observation of very large and steep internal waves of elevation near the Massachusetts coast. Geophys. Res. Lett., 31, L22307, https://doi.org/10.1029/ 2004GL021052.

Shroyer, E. L., J. N. Moum, and J. D. Nash, 2009: Observations of polarity reversal in shoaling nonlinear internal waves. J. Phys. Oceanogr., 39, 691-701, https://doi.org/10.1175/2008JPO3953.1.

Stanton, T. P., and L. A. Ostrovsky, 1998: Observations of highly nonlinear internal solitons over the continental shelf. Geophys. Res. Lett., 25, 2695-2698, https://doi.org/10.1029/98GL01772.

Staquet, C., and J. Sommeria, 2002: Internal gravity waves: from instabilities to turbulence. Annu. Rev. Fluid Mech., 34, 559-593, https://doi.org/10.1146/annurev.fluid.34.090601.130953.

Stastna, M., and K. G. Lamb, 2002: Large fully nonlinear internal solitary waves: the effect of background current. Phys. Fluids, 14, 2987-2999, https://doi.org/10.1063/1.1496510.
Talipova, T. G., E. N. Pelinovsky, K. G. Lamb, R. Grimshaw, and P. Holoway, 1999: Cubic nonlinearity effects in the propagation of intense internal waves. Dokl. Earth Sci., 365, 241-244.

Woodson, C. B., 2018: The fate and impact of internal waves in nearshore ecosystems. Annu. Rev. Mar. Sci., 10, 421-441, https://doi.org/10.1146/annurev-marine-121916-063619.

Xie, J., Y. He, and S. Cai, 2019: Bumpy topographic effects on the transbasin evolution of large-amplitude internal solitary wave in the northern South China Sea. J. Geophys. Res. Oceans, 124, 4677-4695, https://doi.org/10.1029/2018JC014837.

Zhang, S., F. Qiu, J. Zhang, J. Shen, and J. Cha, 2018: Monthly variation on the propagation and evolution of internal solitary waves in the northern South China Sea. Cont. Shelf Res., 171, 21-29, https://doi.org/10.1016/j.csr.2018.10.014.

Zhang, X., X. Huang, Z. Zhang, C. Zhou, J. Tian, and W. Zhao, 2018: Polarity variations of internal solitary waves over the continental shelf of the northern South China Sea: Impacts of seasonal stratification, mesoscale eddies, and internal tides. J. Phys. Oceanogr., 48, 1349-1365, https://doi.org/10.1175/ JPO-D-17-0069.1.

Zhao, Z., V. V. Klemas, Q. Zheng, and X.-H. Yan, 2003: Satellite observation of internal solitary waves converting polarity. Geophys. Res. Lett., 30, 1988, https://doi.org/ 10.1029/2003GL018286.

,,,--- X. Li, and X.-H. Yan, 2004: Estimating parameters of a two-layer stratified ocean from polarity conversion of internal solitary waves observed in satellite SAR images. Remote Sens. Environ., 92, 276-287, https://doi.org/10.1016/ j.rse.2004.05.014. 